\title{
Tuned mass-damper-inerter (TMDI) for suppressing edgewise vibrations of wind turbine blades
}

\author{
Zili Zhang ${ }^{\mathrm{a}}$, Breiffni Fitzgerald ${ }^{\mathrm{b}, *}$ \\ ${ }^{a}$ Department of Engineering, Aarhus University, 8000 Aarhus, Denmark \\ ${ }^{b}$ School of Engineering, Trinity College Dublin, Ireland
}

\begin{abstract}
This paper proposes the use of a tuned mass-damper-inerter (TMDI) for the mitigation of edgewise blade vibrations in wind turbines. The hollow nature of the wind turbine blades is utilized to install a TMDI at a location close to the tip of each blade. A flexible multi-modal offshore wind turbine model is developed in order to study the dynamics of wind turbine blade vibrations. Uncontrolled, TMD controlled and TMDI controlled models are derived. These models are developed using the Euler-Lagrangian approach and lead to time-varying systems with the possibility of negative damping. Closed-form expressions for the optimal tuning and damping ratios of blade-mounted TMDIs are derived. Numerical simulations are then presented to demonstrate the performance of the TMDI controlled blades. The results show that TMDIs can control edgewise vibrations in wind turbine blades while requiring significantly less damper stroke than classical TMDs. The inclusion of the inerter in the damper had a significant effect on the damper stroke with reductions of up to 55\% demonstrated. These impressive reductions in damper stroke come at the cost of very slightly increased blade vibration as compared to the TMD controlled case. This is a trade-off that must be considered in the design of a wind turbine.
\end{abstract}

Keywords: TMDI; Inerter; Wind Turbine; Vibration Control; closed-form solution; optimal design

\section{Introduction}

Tuned mass dampers (TMDs) are one of the simplest and most effective devices that have been developed to control the response of civil engineering structures. TMDs have traditionally been used to mitigate the wind [1], seismic [2] or wave [3] induced response of large, flexible structures. TMDs consist of a small oscillator (secondary system) attached to the structure (primary system) which is subjected to dynamic loads. The fundamental idea behind the TMD is to transfer mechanical energy from the primary system to the secondary system, such that the primary system only performs moderate vibrations. The secondary system is allowed to undergo vibrations, which are dissipated by the damper. The tuning of the mass damper refers to the selection of the parameters of the secondary system (mass, damping and stiffness) in a way

${ }^{*}$ Corresponding author, Tel: +35318961146

Email addresses: zili_zhang@eng.au.dk (Zili Zhang), Breiffni.Fitzgerald@tcd.ie (Breiffni Fitzgerald) 
such that maximum mechanical energy is transferred from the primary system to the secondary system for any given dynamic loading [4]. The concept of using a TMD to control vibrations dates back over one hundred years to Frahm's original damper [5]. The frequency of the damper was tuned to the frequency of the excitation. The damper absorbed the energy from the excitation and underwent vibration, the structure experienced no vibration. Frahm's device had no inherent damping and the performance was poor if the excitation frequency differed marginally from the device's natural frequency. Since Frahm, many investigations have been carried out to optimize and improve the performance of mass dampers. Ormondroyd and DenHartog [6] introduced damping into Frahm's device and observed a noticeable improvement. DenHartog [7] later developed simple expressions for optimal tuning and damping ratios for TMDs. Fujino and Abe [8] derived optimal formulas for designing a TMD for various types of loading. These formulae were expressed as functions of the mass ratio, tuning ratio and damping ratio of the TMD. Rana and Soong [9] studied the effects of tuning of TMDs. The effect of mistuning was investigated and the authors enhanced the understanding of some important characteristics of TMDs. Ghosh and Basu [10] derived a closed form expression for the optimum tuning ratio of a TMD based on the fixed-point theory of DenHartog [7]. Krenk [11] characterized the damping properties of TMDs by dynamic amplification analysis as well as identification of the locus of the complex natural frequencies. Using frequency analysis Krenk derived an optimal TMD damping parameter which is an improvement on the classical value derived by DenHartog [7].

Over the past decade or so there have also been developments and innovations in TMDs. The concept of an 'inerter' was proposed by Smith [12]. Smith defined this device as a two-node, one-port mechanical element with the property that the force applied at the nodes is proportional to the relative acceleration between them - the so called 'inertance' is the constant of proportionality. There are many parasitic effects that are typically neglected in this idealized inerter model, these have been observed in laboratory experiments and in operational inerter devices, such as ratcheting, backlash and friction phenomena [13]. These parasitic effects can be largely mitigated by using the recently proposed fluid inerter [14], which is less popular than the mechanical inerter but has good potential for vibration control. The fluid inerter can reduce the parasitic effects that are more pronounced in mechanical devices and also has greater inherent damping due to the fluid viscosity and density [15]. However, the mechanical inerter is more popular to date in the literature and in practice.

Recent studies have proposed novel TMD configurations incorporating inerters. Most of these studies seek to improve upon the performance of classical TMDs by utilising the theoretical mass amplification effect of the inerter. This mass amplification effect is present when one terminal of the inerter is connected to the ground, referred to in the literature as 'grounded'. When grounded, one advantage of an inerter-based damper is to split the inertial properties in a different manner compared to a traditional TMD. The performance of the inerter-based damper can be improved by using a small mass ratio alongside a large inertance ratio, which can be practically achieved by exploiting the mass-amplification effect of the inerter. It has been demonstrated that an inerter-based damper with smaller mass ratio can lead to higher vibration control than a TMD with higher mass ratio, provided a reasonable choice of the inertance ratio is made and the inerter is grounded [16, 17]. It has also been demonstrated for grounded inerter-based dampers, that using larger inertance ratios can also improve the robustness of the system and reduce deterioration of damper performance due to detuning effects $[18,19]$.

Inerter based TMDs often employ a gearing (via rack and pinion systems or hydraulic devices) that provides an effective mass for the damper much greater than its actual mass. In theory, since a TMD's performance is largely related to its mass ratio, inerter based TMDs can achieve 
greater vibration reductions due to the increased effective mass ratio provided by the inerter. Ikago et al. [20] proposed the tuned viscous mass damper (TVMD), a new structural control device for seismic applications. The device used an inerter (ball-screw mechanism) to achieve a mass amplification effect. Closed-form expressions were derived for optimal device parameters and experimental verification was provided by shake table tests. Garrido et al. [21] developed a new passive vibration control device, the rotational inertia double tuned mass damper (RIDTMD). The RIDTMD consists of a conventional TMD in which the typical viscous damper is replaced with a tuned viscous mass damper. The RIDTMD is more effective than a TMD having the same mass ratio and has a wider suppression band. The tuned mass-damper-inerter (TMDI) was proposed by Marian and Giaralis [22]. This device utilises the mass amplification effect of the inerter, in this case a two-terminal flywheel device, to improver upon the classical TMD performance. Optimal TMDI parameters were derived in closed-form as functions of the TMD mass and the inerter constant. The authors concluded that incorporating an inerter in the damper can either replace part of the TMD vibrating mass to achieve a lightweight damper, or improve upon the performance of the classical TMD for a given TMD mass. Giaralis and Taflanidis [23] considered reliability based optimal design of building structures with TMDIs installled under seismic loading. The TMDI's frequency, damping ratio, and inertance parameters were optimised subject to constraints on floor accelerations and TMDI stroke. The enhanced performance of the TMDI over the classical TMD especially for relatively small additional attached mass was reported. Lazar et al. [24] also proposed a new mass damper device incorporating an inerter. The focus of the study was on vibration suppression in base excited structures. The authors demonstrated the performance improvement achieved by using an inerter device instead of a classical TMD. Lazar et al. [25] also investigated the use of a tuned-inerter-damper (TID) for vibration suppression in cables. The device was optimised and a practical design method developed. Hu and Chen [26] optimised inerter-based dynamic vibration absorbers (IDVAs). IDVAs are developed by replacing the damper in the traditional dynamic vibration absorber (DVA) with an inerter. Four different IDVAs configurations were proposed by adding an inerter together with a spring to the DVA, and significant improvement for both the $\mathrm{H}_{\infty}$ and $\mathrm{H}_{2}$ performances is obtained. A recent study by Shen et al. [27] on the application of TIDs to civil engineering structures concluded that TIDs outperform conventional TMDs on response control of primary structures subjected to dynamic loads, since the former will not increase the total energy input into the system but the latter will. TID's also have the advantage of achieving very large inertance-to-mass ratio without increasing space demand (or damper stroke).

For the past decade the wind energy industry has been applying the methodologies and technologies developed in the field of structural control to reduce vibrations in wind turbines. The reduction of the blade response is a critical concern of manufacturers and infrastructure operators and owners. Modern wind turbine blades are very long (can now be $>80 \mathrm{~m}$ ) slender structures that are subjected to large dynamic loads. Blades are manufactured from lightweight high-strength materials and as such they are very flexible and lightly damped. Due to these structural characteristics, they are very susceptible to wind induced vibrations and they may undergo significant vibration during operation under turbulent aerodynamic loads. Blade vibrations may slow down wind energy conversion to electrical power and reduce the fatigue life of the turbine structure [28]. Dueñas-Osorio and Basu [29] investigated wind turbine unavailability as a function of wind-induced vibrations of the turbine. Blade vibration can lead to the malfunction of acceleration-sensitive equipment housed in the nacelle of the wind turbine, resulting in reduced annual wind turbine availability. Blade vibration issues may have a significant impact on the lifetime of the structural components, and even on the overall integrity of the structural system. 
These impacts will have associated implications for the cost of wind power. Therefore, there is now increasing interest on reducing the harmful effects of mechanical vibration on wind turbine blades in the wind energy industry and structural control of wind turbine blades is now an active area of research.

Passive devices such as Tuned Liquid Dampers [30, 31]; Tuned Liquid Column Dampers [28]; Circular Liquid Dampers [32]; and Roller Dampers [33] have been proposed for vibration control of wind turbine blades. Passive tuned mass dampers (TMDs) have been investiagted in many studies [34, 35]. It should be noted that liquid dampers have been found to perform well in experimental studies on wind turbine blades [36] and they do not suffer from stroke limitation issues inherent in traditional mass damper devices. Semi-active devices such as variable stiffness TMDs have been developed for wind turbine applications with algorithms for flapwise blade vibrations [37], edgewise blade vibrations [38] and tower vibrations [39] developed. Active control devices including active mass dampers have also been investigated [40, 41, 42, 43]. Active control devices have also been proposed for non-resonant vibration control of wind turbine blades utilising active tendons [44] or cables [45]. Active individual blade pitch control has also been proposed as a method of suppressing non-resonant vibrations [46, 47, 48, 49]. Wind turbine manufacturers are now installing dampers inside wind turbine blades. However, there remain challenges such as the limited space available inside the hollow blade and concerns about large damper mass required to suppress vibrations. The tuned mass damper inerter (TMDI) offers an alternative choice for the designer. Using a TMDI, the damper stroke required for vibration control may be reduced.

Recent literature has proposed the use of inerter-based dampers in wind turbines. All of the literature so far concerns installation of these devices in the nacelle or tower with no studies investigating their application in wind turbine blades. Hu et al. [50] applied an inerter-based damper to a barge type floating offshore wind turbine. The inerter consisted of a parallel connection of a spring, a damper, and an inerter-based network. The damper parameters were optimised and performance improvement over a traditional TMD was noted. Zhang et al. [51] developed the tuned parallel inerter mass system (TPIMS) for vibration control of wind turbine towers under seismic loads. Damper parameters were again optimised and the primary structure's response was reduced compared to results with a classical TMD of the same mass ratio. Ma et al. [52] proposed an inerter-based device called the tuned heave plate inerter (THPI). The device was applied to semi submersible platforms which are used as a floating foundation solution for some offshore wind turbines. The mass amplification effect of the inerter was employed to amplify the effective damper mass and improvements were thus achieved over conventional structural control schemes. Ma et al. [53] also developed another novel damper - the hydraulic rotational inertia damper (RID), to mitigate heave motions of semi submersible platforms. Sarkar and Fitzgerald [54] recently proposed the use of a TMDI for vibration control of spar type floating offshore wind turbine towers.

In this paper, TMDIs are proposed to reduce edgewise vibrations in wind turbine blades. The hollow nature of the wind turbine blades is utilized to install a TMDI at a location close to the tip of each blade. A flexible offshore wind turbine model is developed in order to study the dynamics of wind turbine blade vibrations, including the coupling between the edgewise and the flapwise modes of the blades. A multi-modal representation of the flexible elements (the three blades and the tower) is adopted. The equations of motion are derived by taking into account the blade-tower interaction. Uncontrolled, TMD controlled and TMDI controlled models are derived. These models are developed using the Euler-Lagrangian approach and lead to time-varying systems with the possibility of negative damping. Closed-form expressions for 
the optimal tuning parameters of blade-mounted TMDIs are derived. Numerical simulations are then presented to demonstrate the performance of the TMDI controlled blades. The results indicate encouraging prospects for the use of TMDIs in the control of vibrations of wind turbine blades with implications for industry.

\section{Modeling}

\subsection{3-DOF aeroelastic wind turbine model}

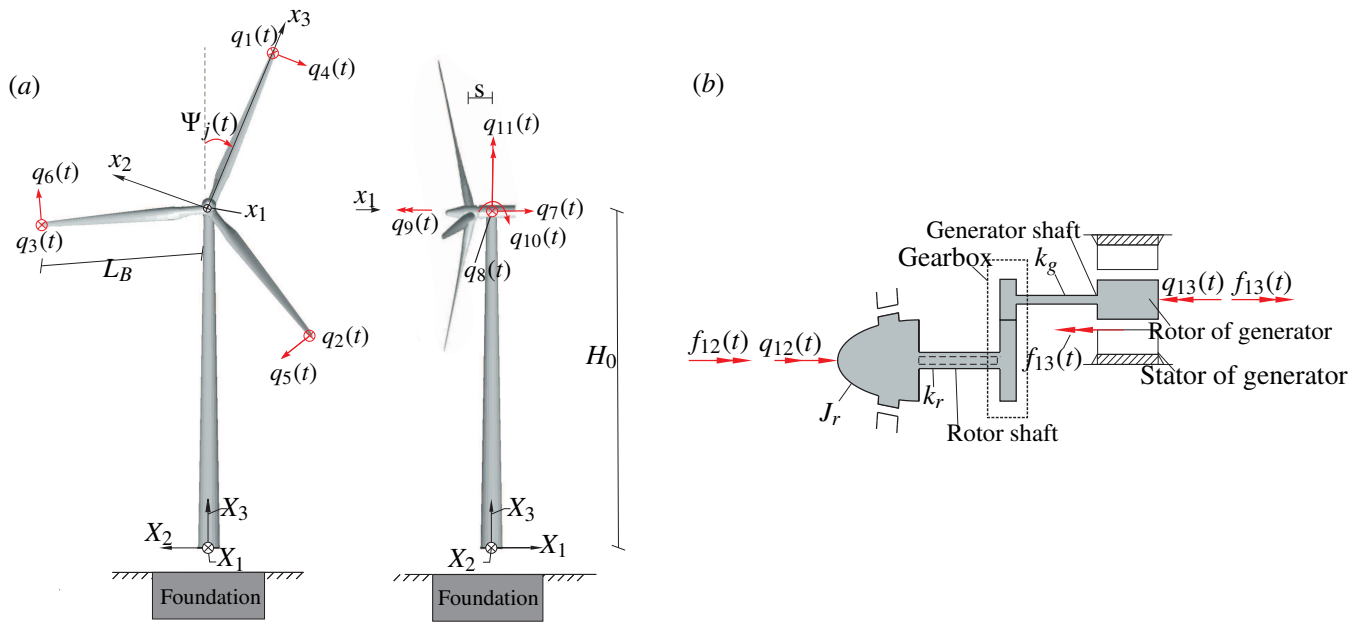

Figure 1: The 13-DOF model. (a) DOFs of the blades and tower. (b) DOFs of the flexible drivetrain.

A 13-degree-of-freedom (13-DOF) aeroelastic wind turbine model developed using a modal approach together with analytical dynamics method $[33,55]$ is briefly presented in this subsection, which will be used in the later section for evaluating the performance of the optimal TMDI in a highly-coupled wind turbine system. This model possesses the most important features of a wind turbine system, i.e. time-dependent system matrices, coupled tower-blade-drivetrain vibrations as well as nonlinear aeroelasticity.

Figure 1 shows the definitions of DOFs of this 13-DOF model as well as the coordinate systems. Each blade is modelled in a moving local $\left(x_{1}, x_{2}, x_{3}\right)$-coordinate system as a rotating Bernoulli-Euler beam (with both centrifugal stiffening and centripetal softening effects considered), and is associated with 2 DOFs representing the flapwise vibration and edgewise vibration, respectively. The tower and the flexible drivetrain are modeled in the fixed global $\left(X_{1}, X_{2}, X_{3}\right)$ coordinate system, as a Bernoulli-Euler beam and a St. Venant torsional rod, respectively. The tower vibration is described by 2 translational DOFs $q_{7}(t)$ and $q_{8}(t)$, and 3 rotational DOFs $q_{9}(t)$, $q_{10}(t)$ and $q_{11}(t)$. Finally, the torsional motion of the flexible drivetrain is described by 2 DOFs $q_{12}(t)$ and $q_{13}(t)$, signifying the deviations of the rotational angles at the hub and at the generator from the nominal rotational angles $\Omega_{0} t$ and $N \Omega_{0} t$, respectively, where $\Omega_{0}$ is the nominal rotational speed and $N$ is the gear ratio.

The equations of motion of the 13-DOF model are obtained by substituting the formulated kinetic and potential energies of the complete wind turbine system into the Euler-Lagrange equation. For obtaining the aerodynamic loads, rotational sampled turbulence is generated using 
Taylor's hypothesis of frozen turbulence [55] together with a first-order autoregressive model [56]. The corrected blade element momentum (BEM) method is then employed to calculate the aerodynamic loads along each blade. Nonlinear quasi-static aeroelasticity is considered by incorporating the local deformation velocities of the blade cross-sections into angle of attack calculation. Furthermore, a collective pitch controller (with time delay modeled by a first-order filter) and a generator torque controller are also included in the 13-DOF model.

\subsection{Definition of the problem: TMDI in a rotating blade}

Figure 2 shows the schematic representation of a rotating blade equipped with a tuned massdamper-inerter (TMDI). The edgewise vibration of the blade is described in the moving $\left(x_{1}, x_{2}, x_{3}\right)$ coordinate system. For each blade (assumed to be identical), the mass per unit length and the bending stiffness in the edgewise direction are denoted $\mu\left(x_{3}\right)$ and $E I\left(x_{3}\right)$, respectively. The rotation of each blade is assumed to take place with a constant rotational speed $\Omega$. For modern multi-megawatt pitch-regulated wind turbines, the rotational speed is constant (slightly oscillating around this mean value due to turbulence) when the mean wind speed is between the rated wind speed and the cut-out wind speed due to the functioning of the pitch controller [57]. Below rated, the pitch controller is deactivated, and the rotational speed is dependent on the mean wind speed. Hence, in general the rotational speed can be considered constant for a given wind speed. Furthermore, $\Omega$ here is merely a system parameter, which appears in the closed-form expressions for the optimal frequency tuning and damping ratio of the blade-mounted TMDI, as will be shown below. This will provide a basis for the possible semi-active control when $\Omega$ changes due to the change of wind speed.

The azimuthal angle $\Psi_{j}(t)$ for blade $j$ is thus given by:

$$
\Psi_{j}(t)=\Omega t+\frac{2 \pi}{3}(j-1), \quad j=1,2,3
$$

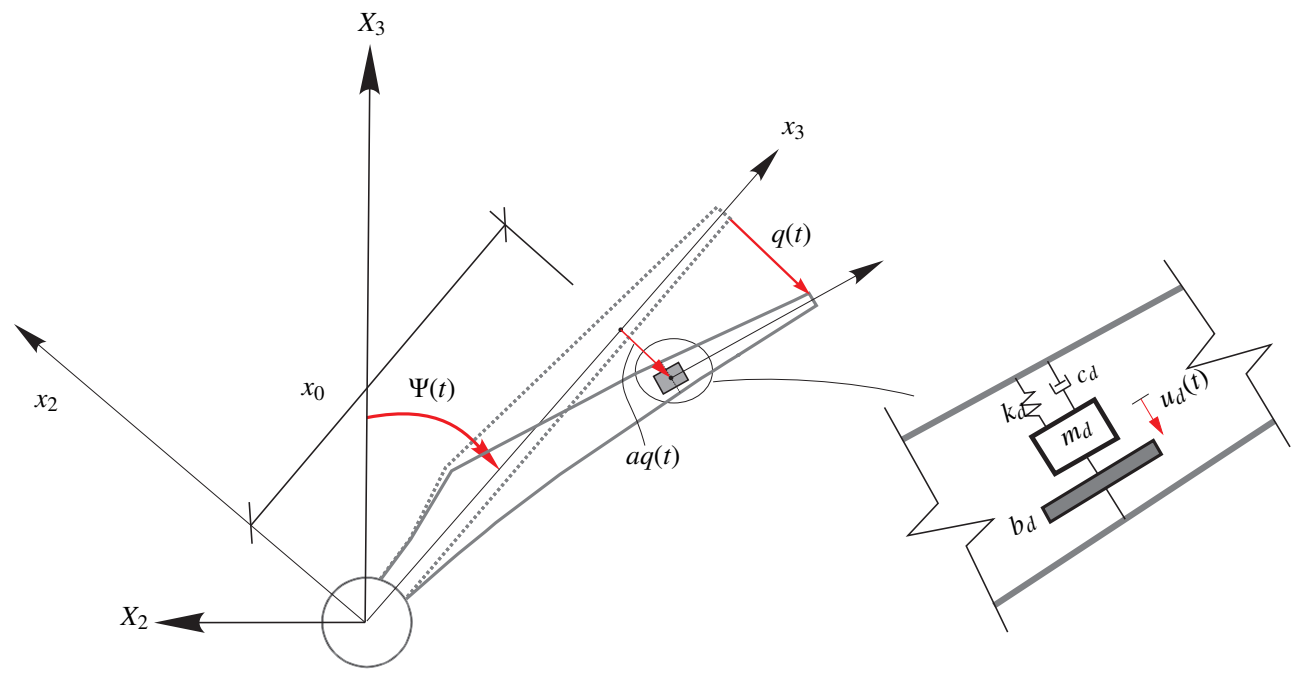

Figure 2: Definition of coordinate systems, geometry and degrees of freedom.

In the following, only blade $j=1$ is considered since all three blades have identical geometrical and structural parameters. Hence, $\Psi_{j}(t)=\Psi(t)=\Omega t$. The edgewise vibration of the blade 
is described by the local degree of freedom $q(t)$, representing the tip displacement in the negative $x_{2}-$ direction. The local edgewise displacement field $u_{2}\left(x_{3}, t\right)$ of the rotating blade in the positive $x_{2}-$ direction can be described by $\mathrm{q}(\mathrm{t})$ as:

$$
u_{2}\left(x_{3}, t\right)=-\Phi\left(x_{3}\right) q(t)
$$

where $\Phi\left(x_{3}\right)$ is the fundamental edgewise eigenmode of the blade, which is normalized to 1 at the blade tip.

Assuming the TMDI to be installed at the coordinate $x_{3}=x_{0}$, the local displacement of the blade at this position with sign definition in Figure $\mathrm{X}$ is written as:

$$
u_{2}\left(x_{0}, t\right)=-a q(t)
$$

where $a=\Phi\left(x_{0}\right) . a$ is an important design parameter for TMDI representing the influence of the damper location.

The TMDI is a device which combines a conventional/classical tuned mass damper (TMD) with an inerter. The conventional TMD is installed inside the blade at the position $x_{0}$, which consists a block mass $m_{d}$ attached to the primary structure via a linear spring of stiffness $k_{d}$ and a viscous damper with damping coefficient $c_{d}$. One end of the inerter (with inertance $b_{d}$ ) is connected to the TMD, and the other end is connected to the blade structure. The physical mass of the inerter is negligible compared to the masses $m_{d}$ and $b_{d}$. The displacement of the TMD block mass relative to the deformed blade is denoted $u_{d}(t)$, as shown in Figure 1. Therefore, $q(t)$ and $u_{d}(t)$ make up the degrees of freedom of the 2-DOF blade-TMDI system.

\subsection{Modeling of the 2-DOF blade-TMDI system}

The velocity components of the blade in the moving $\left(x_{2}, x_{3}\right)$-coordinate system can be written as:

$$
\left.\begin{array}{l}
v_{2}\left(x_{3}, t\right)=-\Omega x_{3}-\Phi\left(x_{3}\right) \dot{q}(t) \\
v_{3}\left(x_{3}, t\right)=-\Omega \Phi\left(x_{3}\right) q(t)
\end{array}\right\}
$$

The components of the position vector and velocity vector of the TMD block mass in the fixed global $\left(X_{2}, X_{3}\right)$-coordinate system are given by:

$$
\begin{aligned}
& X_{2, d}(t)=-x_{0} \sin \Psi-a q \cos \Psi-u_{d} \cos \Psi \\
& \left.X_{3, d}(t)=x_{0} \cos \Psi-a q \sin \Psi-u_{d} \sin \Psi\right\} \\
& \left.V_{2, d}(t)=-\left(x_{0} \Omega+a \dot{q}+\dot{u}_{d}\right) \cos \Psi+\left(a q+u_{d}\right) \Omega \sin \Psi\right) \\
& \left.V_{3, d}(t)=-\left(x_{0} \Omega+a \dot{q}+\dot{u}_{d}\right) \sin \Psi-\left(a q+u_{d}\right) \Omega \cos \Psi\right\}
\end{aligned}
$$

The total kinetic energy of the system (i.e. one blade and one TMDI including the block mass and the inerter) becomes:

$$
\begin{aligned}
T(t) & =\frac{1}{2} \int_{0}^{L} \mu\left(x_{3}\right)\left(v_{2}^{2}\left(x_{3}, t\right)+v_{3}^{2}\left(x_{3}, t\right)\right) d x_{3}+\frac{1}{2} m_{d}\left(V_{2, d}^{2}(t)+V_{3, d}^{2}(t)\right)+\frac{1}{2} b_{d} \dot{u}_{d}^{2} \\
& =\frac{1}{2} m_{0}\left(\dot{q}^{2}+\Omega^{2} q^{2}\right)+m_{1} \Omega \dot{q}+\frac{1}{2} \Omega^{2} m_{2}+\frac{1}{2} m_{d}\left[\left(x_{0} \Omega+a \dot{q}+\dot{u}_{d}\right)^{2}+\left(a q \Omega+u_{d} \Omega\right)^{2}\right]+\frac{1}{2} b_{d} \dot{u}_{d}^{2}
\end{aligned}
$$


where $m_{0}=\int_{0}^{L} \mu\left(x_{3}\right) \Phi^{2}\left(x_{3}\right) d x_{3}$ denotes the modal mass of the blade. Further, $m_{1}=\int_{0}^{L} \mu\left(x_{3}\right) x_{3} \Phi\left(x_{3}\right) d x_{3}$, $m_{2}=\int_{0}^{L} \mu\left(x_{3}\right) x_{3}^{2} d x_{3}$. The total potential energy of the system is:

$$
U(t)=m_{d} g\left(x_{0} \cos \Psi-a q \sin \Psi-u_{d} \sin \Psi\right)+\frac{1}{2} k_{0} q^{2}+\frac{1}{2} k_{d} u_{d}^{2}
$$

where $g$ is the gravitational acceleration. $k_{0}$ denotes the modal stiffness of the blade including the geometric stiffness effect from centrifugal accelerations, which is expressed as:

$$
k_{0}=\int_{0}^{L}\left(E I\left(x_{3}\right)\left(\frac{d^{2} \Phi\left(x_{3}\right)}{d x_{3}^{2}}\right)^{2}+F\left(x_{3}\right)\left(\frac{d \Phi\left(x_{3}\right)}{d x_{3}}\right)^{2}\right) d x_{3}
$$

with $F\left(x_{3}\right)=\Omega^{2} \int_{x_{3}}^{L} \mu(\xi) \xi d \xi$ being the centrifugal axial force per unit length along the blade. Therefore, the fundamental angular eigenfrequency of the blade is obtained as:

$$
\omega_{0}=\sqrt{\frac{k_{0}}{m_{0}}}
$$

Using Eqs. (7), (8), the equations of motion of the 2-DOF blade-TMDI system can be obtained from the stationarity conditions of the Euler-Lagrange equations [58]:

$$
\begin{gathered}
\frac{d}{d t}\left(\frac{\partial T}{\partial \dot{q}}\right)-\frac{\partial T}{\partial q}+\frac{\partial U}{\partial q}=f(t)-c_{0} \dot{q} \Rightarrow \\
\left(m_{0}+a^{2} m_{d}\right) \ddot{q}+a m_{d} \ddot{u}_{d}+c_{0} \dot{q}+\left[k_{0}-\left(m_{0}+a^{2} m_{d}\right) \Omega^{2}\right] q-a m_{d} \Omega^{2} u_{d}=f(t)+a m_{d} g \sin \Psi \\
\frac{d}{d t}\left(\frac{\partial T}{\partial \dot{u}_{d}}\right)-\frac{\partial T}{\partial u_{d}}+\frac{\partial U}{\partial u_{d}}=-c_{d} \dot{u}_{d} \Rightarrow \\
a m_{d} \ddot{q}+\left(m_{d}+b_{d}\right) \ddot{u}_{d}+c_{d} \dot{u}_{d}-a m_{d} \Omega^{2} q+\left(k_{d}-m_{d} \Omega^{2}\right) u_{d}=m_{d} g \sin \Psi
\end{gathered}
$$

where $f(t)$ denotes the modal load on the blade due to turbulence and the gravity, which is calculated using the more sophisticated 13-DOF aeroelastic wind turbine model introduced in subsetion 2.1. $c_{0}=2 \zeta_{0} m_{0} \omega_{0}$ indicates the modal damping coefficient of the primary structure, with $\zeta_{0}$ being the corresponding modal damping ratio.

Eqs. (11) and (12) can be combined into matrix form:

$$
\begin{array}{r}
{\left[\begin{array}{cc}
m_{0}+a^{2} m_{d} & a m_{d} \\
a m_{d} & m_{d}+b_{d}
\end{array}\right]\left[\begin{array}{c}
\ddot{q} \\
\ddot{u}_{d}
\end{array}\right]+\left[\begin{array}{cc}
c_{0} & 0 \\
0 & c_{d}
\end{array}\right]\left[\begin{array}{c}
\dot{q} \\
\dot{u}_{d}
\end{array}\right]+\left[\begin{array}{cc}
k_{0}-\left(m_{0}+a^{2} m_{d}\right) \Omega^{2} & -a m_{d} \Omega^{2} \\
-a m_{d} \Omega^{2} & k_{d}-m_{d} \Omega^{2}
\end{array}\right]\left[\begin{array}{c}
q \\
u_{d}
\end{array}\right]} \\
=\left[\begin{array}{c}
f(t)+a m_{d} g \sin \Psi \\
m_{d} g \sin \Psi
\end{array}\right]
\end{array}
$$

In the case of a classical TMD, the equations of motion of the 2-DOF blade-TMD system is obtained directly from Eq. (13) with $b_{d}=0$. 


\section{Optimal tuning of the bladed-mounted TMDI}

For deriving closed-form expressions of the optimal tuning of the blade-mounted TMDI, the following simplifications are made: (a) The damping of the primary structure is ignored, i.e. $c_{0}=0$. This makes sense because of the inherently low structural damping of the blade and the negligible aerodynamic damping of the edgewise mode. (b) The two terms $a m_{d} g \sin \Psi$ and $m_{d} g \sin \Psi$ in the load vector are negligible comparing with the term $f_{0}$, since the TMD block mass is insignificant. On the other hand, the performance of the optimally designed TMDI will be justified using the original equations of motion through time-domain simulations.

\subsection{Normalized amplitude and dynamic amplification}

The responses $q(t), u_{d}(t)$ and the load $f(t)$ are represented in terms of harmonic components with angular frequency $\omega$ :

$$
q(t)=q_{0} e^{i \omega t}, \quad u_{d}(t)=u_{d, 0} e^{i \omega t}, \quad f(t)=f_{0} e^{i \omega t}
$$

where $q_{0}, u_{d, 0}$ and $f_{0}$ are the complex amplitudes. $i=\sqrt{-1}$ is the complex unit. Harmonic load is assumed in order to derive the closed-form expressions for the optimal frequency tuning and damping ratio of the blade-mounted TMDI. On the other hand, the performance of the optimized TMDI in a rotating blade will be evaluated using time-domain simulations with turbulent wind loads as the inputs, as will be shown in subsections 4.3 and 4.4 .

Substituting Eq. (14) into Eq. (13), the normalized amplitude of the structural response is given by:

$$
\frac{q_{0}}{f_{0} / k_{0}}=\frac{A+2 i \zeta_{d} B}{C+2 i \zeta_{d} D}
$$

where

$$
\begin{aligned}
& A=(1+\gamma) \omega_{0}^{2}\left(\omega_{d}^{2}-\frac{\Omega^{2}}{1+\gamma}-\omega^{2}\right) \\
& B=(1+\gamma) \omega_{0}^{2} \omega_{d} \omega \\
& C=\left(1+\gamma+a^{2} \beta\right) \omega^{4}-\left[(1+\gamma) \omega_{0}^{2}+(1+\gamma)\left(1+a^{2} \mu\right) \omega_{d}^{2}-\left(2+\gamma+a^{2} \beta\right) \Omega^{2}\right] \omega^{2}+\left[(1+\gamma) \omega_{0}^{2} \omega_{d}^{2}-\left(\omega_{0}^{2}+(1+\gamma)\left(1+a^{2} \mu\right) \omega_{d}^{2}\right) \Omega^{2}+\Omega^{4}\right] \\
& D=(1+\gamma) \omega_{d}\left[\left(\omega_{0}^{2}-\left(1+a^{2} \mu\right) \Omega^{2}\right) \omega-\left(1+a^{2} \mu\right) \omega^{3}\right]
\end{aligned}
$$

In Eqs. (15) and (16), the following normalized parameters of the TMDI have been introduced:

$$
\mu=\frac{m_{d}}{m_{0}}, \quad \beta=\frac{b_{d}}{m_{0}}, \quad \gamma=\frac{b_{d}}{m_{d}}=\frac{\beta}{\mu}, \quad \omega_{d}=\sqrt{\frac{k_{d}}{m_{d}+b_{d}}}, \quad \zeta_{d}=\frac{c_{d}}{2 \sqrt{k_{d}\left(m_{d}+b_{d}\right)}}
$$

where $\mu$ and $\beta$ are the mass ratio and inertance ratio, respectively. $\omega_{d}$ and $\zeta_{d}$ are the angular natural frequency and damping ratio of the decoupled TMDI, respectively. The corresponding normalized parameters of a classical TMD are obtained simply by setting $b_{d}=0$.

The dynamic amplification of the structural response is the absolute value (module) of Eq. (15), which is used as the basis for determining the optimal parameters of the blade-mounted TMDI in the following subsections. 
Similarly, the normalized amplitude of the relative TMD mass block motion is expresses as:

$$
\frac{u_{d, 0}}{f_{0} / k_{0}}=\frac{a \omega_{0}^{2} \omega^{2}+a \omega_{0}^{2} \Omega^{2}}{C+2 i \zeta_{d} D}
$$

Furthermore, the normalized form of the so-called frequency equation (or characteristic polynomial) can be obtained by letting the denominator of Eq. (15) (or Eq. (18)) be zero:

$$
C+2 i \zeta_{d} D=0
$$

which essentially determines the complex natural frequencies of the free vibration modes of the blade-TMDI system. An important case is when $\zeta_{d} \rightarrow \infty$, which implies that the viscous damper completely constrains the relative TMD motion $u_{d}(t)$, i.e. the TMDI is locked. In this case, the solution to Eq. (19) becomes real, which represents the natural frequency of the blade with a locked TMDI:

$$
\omega_{\infty}=\sqrt{\frac{\omega_{0}^{2}}{1+a^{2} \mu}-\Omega^{2}}
$$

This natural frequency $\omega_{\infty}$ will be used as a reference frequency when deriving the closedform expression of the optimal TMDI damping ratio $\zeta_{d}$.

As for the case of classical TMD, $\omega_{\infty}$ turns out to be exactly the same as in Eq. (20). This makes sense because when the block mass $m_{d}$ is locked, the inertance $b_{d}$ effectively becomes zero (and thus the TMDI is reduced to TMD) since the relative acceleration between the two terminals of the inerter is zero.

For optimal design of the blade-mounted TMDI, the optimal values of $\omega_{d}$ and $\zeta_{d}$ need to be determined when the damper location $x_{0}$, the mass ratio $\mu$ and the inertance ratio $\beta$ are prescribed.

\subsection{Optimal frequency tuning}

According to the classical TMD theory [7, 11], around $\omega_{0}$ there are two excitation frequencies $\omega_{M}$ and $\omega_{N}$ where the dynamic amplifications are independent of the TMD damping ratio $\zeta_{d} . \omega_{M}$ and $\omega_{N}$ are denoted the neutral frequencies or the fixed-point frequencies. It turns out that the fixed-point frequencies also exist for the present case of a rotating blade equipped with a TMDI. The classical TMD theory provides the optimal frequency-tuning criterion, i.e. the optimal frequency $\omega_{d}$ is chosen to ensure the equal dynamic amplification at the two fixed-point frequencies $\omega_{M}$ and $\omega_{N}$. This criterion is also employed in the present paper.

At $\omega_{M}$ and $\omega_{N}$, the dynamic amplifications are independent of the TMDI damping ratio $\zeta_{d}$. This leads to the following equation by the use of Eq. (15):

$$
\frac{A^{2}}{C^{2}}=\frac{B^{2}}{D^{2}} \quad \Rightarrow \quad A D= \pm B C
$$

Substituting Eq. (16) into Eq. (21) and recognizing that the plus sign in Eq. (21) only leads to the trivial solution $\omega=0$, the polynomial fulfilled by $\omega=\omega_{M}$ or $\omega=\omega_{N}$ is obtained:

$$
\begin{array}{r}
\left(2+a^{2} \mu+2 \gamma+2 a^{2} \beta\right) \omega^{4}-2\left[(1+\gamma) \omega_{0}^{2}+(1+\gamma)\left(1+a^{2} \mu\right) \omega_{d}^{2}-\left(2+a^{2} \mu+\gamma+a^{2} \beta\right) \Omega^{2}\right] \omega^{2} \\
+\left\{2(1+\gamma) \omega_{0}^{2} \omega_{d}^{2}-2\left[\omega_{0}^{2}+(1+\gamma)\left(1+a^{2} \mu\right) \omega_{d}^{2}\right] \Omega^{2}+\left(2+a^{2} \mu\right) \Omega^{4}\right\}=0
\end{array}
$$


from which we directly have the sum of $\omega_{M}^{2}$ and $\omega_{N}^{2}$ :

$$
\omega_{M}^{2}+\omega_{N}^{2}=\frac{2\left[(1+\gamma) \omega_{0}^{2}+(1+\gamma)\left(1+a^{2} \mu\right) \omega_{d}^{2}-\left(2+a^{2} \mu+\gamma+a^{2} \beta\right) \Omega^{2}\right]}{2+a^{2} \mu+2 \gamma+2 a^{2} \beta}
$$

Next, the property of the fixed-point frequencies implies that we can obtain the dynamic amplifications at $\omega_{M}$ and $\omega_{N}$ by letting $\zeta_{d} \rightarrow \infty$ in Eq. (15):

$$
\left|\frac{q_{0}}{f_{0} / k_{0}}\right|_{M, N}=\frac{ \pm 1}{1-\left(1+a^{2} \mu\right)\left(\Omega / \omega_{0}\right)^{2}-\left(1+a^{2} \mu\right)\left(\omega / \omega_{0}\right)^{2}}
$$

To ensure equal dynamic amplification at $\omega_{M}$ and $\omega_{N}$, we have:

$$
\frac{1}{1-\left(1+a^{2} \mu\right)\left(\Omega / \omega_{0}\right)^{2}-\left(1+a^{2} \mu\right)\left(\omega_{M} / \omega_{0}\right)^{2}}=\frac{-1}{1-\left(1+a^{2} \mu\right)\left(\Omega / \omega_{0}\right)^{2}-\left(1+a^{2} \mu\right)\left(\omega_{N} / \omega_{0}\right)^{2}}
$$

from which the second equation for $\omega_{M}^{2}+\omega_{N}^{2}$ is obtained as:

$$
\omega_{M}^{2}+\omega_{N}^{2}=\frac{2 \omega_{0}^{2}-2\left(1+a^{2} \mu\right) \Omega^{2}}{1+a^{2} \mu}
$$

Comparing Eqs. (23) and (26), the closed-form expression of the optimal frequency of the blade-mounted TMDI becomes:

$$
\left(\omega_{d}\right)_{o p t}=\sqrt{\frac{\left(1+\gamma+a^{2} \beta\right) \omega_{0}^{2}-\left(1+a^{2} \mu\right)\left(\gamma+a^{2} \beta\right) \Omega^{2}}{(1+\gamma)\left(1+a^{2} \mu\right)^{2}}}
$$

which turns out to be not only dependent on the mass ratio $\mu$ and the inertance ratio $\beta$, but also dependent on the rotor rotational speed $\Omega$. Once $\left(\omega_{d}\right)_{\text {opt }}$ is determined, the spring stiffness can be calculated as $k_{d}=\left(\omega_{d}\right)_{o p t}^{2}\left(m_{d}+b_{d}\right)$.

As for the classical TMD, the optimal frequency of the blade-mounted TMD can also be obtained using the same procedure, which is given as (without going into details) [59]:

$$
\left(\omega_{d, T M D}\right)_{o p t}=\frac{\omega_{0}}{1+a^{2} \mu}
$$

It is interesting to see that for the classical TMD, the optimal frequency ratio $\frac{\left(\omega_{d, T M D}\right)_{o p t}}{\omega_{0}}$ from Eq. (28) is independent on the rotor rotational speed $\Omega$. By setting $\beta=0$ (and thus $\gamma=0$ ) in Eq. (27), Eq. (27) is exactly reduced to Eq. (28).

Furthermore, substituting the optimal frequency $\left(\omega_{d}\right)_{o p t}$ given by Eq. (27) into Eq. (22), the quadratic equation with $\omega_{M}^{2}$ and $\omega_{N}^{2}$ being the roots becomes:

$$
\omega^{4}-2\left(\frac{\omega_{0}^{2}}{1+a^{2} \mu}-\Omega^{2}\right) \omega^{2}+\left[\frac{2\left(1+\gamma+a^{2} \beta\right) \omega_{0}^{4}}{\left(1+a^{2} \mu\right)^{2}\left(2+a^{2} \mu+2 \gamma+2 a^{2} \beta\right)}-\frac{2 \omega^{2} \Omega^{2}}{1+a^{2} \mu}+\Omega^{4}\right]=0
$$

Solving $\omega_{M}^{2}$ and $\omega_{N}^{2}$ from Eq. (29) and then inserting them into Eq. (24), the dynamic amplification at the fixed-point frequencies $\omega_{M}$ and $\omega_{N}$ can be explicitly written as:

$$
\left|\frac{q_{0}}{f_{0} / k_{0}}\right|_{M, N}=\sqrt{\frac{2+a^{2} \mu+2 \gamma+2 a^{2} \beta}{a^{2} \mu}}
$$


which is seen to be independent of $\Omega$. This to some extent generalizes the fixed-point theory of the classical SDOF-TMD system, i.e. independence of both the damping ratio $\zeta_{d}$ and the rotor rotational speed $\Omega$ are achieved in the present blade-TMDI system.

\subsection{Optimal damping tuning}

In this subsection, closed-form expression of the optimal TMDI damping ratio is derived using the criterion proposed in [11]. The idea is that the optimal TMDI damping ratio is chosen to ensure the dynamic amplifications are equal at three excitation frequencies, $\omega_{M}, \omega_{N}$ and $\omega_{\infty}$. This means $\left(\zeta_{d}\right)_{o p t}$ should be determined in order that the dynamic amplification at $\omega_{\infty}$ is equal to that in Eq. (30).

Substituting Eq. (20) and Eq. (27) into Eq. (15), the normalized amplitude of the structural response at the excitation frequency $\omega=\omega_{\infty}$ becomes:

$$
\frac{q_{0}}{f_{0} / k_{0}}=1-\frac{2 i \zeta_{d}\left(1+a^{2} \mu\right)}{a^{2} \mu \omega_{0}^{2}} \sqrt{\frac{(1+\gamma)\left[\left(1+\gamma+a^{2} \beta\right) \omega_{0}^{4}-\left(1+a^{2} \mu\right)\left(1+2 \gamma+2 a^{2} \beta\right) \omega_{0}^{2} \Omega^{2}+\left(1+a^{2} \mu\right)^{2}\left(\gamma+a^{2} \beta\right) \Omega^{4}\right]}{1+a^{2} \mu}}
$$

which indicates a constant real part regardless of the TMDI parameters. Equalizing the absolute value of Eq. (31) to Eq. (30), the closed-form expression of the optimal TMDI damping ratio becomes:

$$
\left(\zeta_{d}\right)_{o p t}=\sqrt{\frac{1}{2} \frac{\left(a^{2} \mu+a^{2} \beta+a^{4} \mu \beta\right)}{(1+\gamma)\left(1+a^{2} \mu\right)} \frac{\omega_{0}^{4}}{\left(1+\gamma+a^{2} \beta\right) \omega_{0}^{4}-\left(1+a^{2} \mu\right)\left(1+2 \gamma+2 a^{2} \beta\right) \omega_{0}^{2} \Omega^{2}+\left(1+a^{2} \mu\right)^{2}\left(\gamma+a^{2} \beta\right) \Omega^{4}}}
$$

As for the case of classical TMD, the same procedure above can be employed, which results in the following expression of the optimal TMD damping ratio [59]:

$$
\left(\zeta_{d, T M D}\right)_{o p t}=\sqrt{\frac{1}{2} \frac{a^{2} \mu}{1+a^{2} \mu}\left(\frac{\omega_{0}^{2}}{\omega_{0}^{2}-\left(1+a^{2} \mu\right) \Omega^{2}}\right)}
$$

By setting $\beta=0$ (and thus $\gamma=0$ ) in Eq. (32), Eq. (32) is exactly reduced to Eq. (33).

\section{Numerical examples}

Numerical simulations are carried out to demonstrate the performance of the TMDI compared with a classical TMD and the uncontrolled blade response. Data from the NREL $5 \mathrm{MW}$ wind turbine [57] is used to instantiate the structural model of the wind turbine blade. Each blade has a length of $61.5 \mathrm{~m}$ and an overall mass of $17740 \mathrm{~kg}$, with the mass per unit length, the edgewise bending stiffness and the fundamental edgewise mode shape provided by [57]. The modal mass $m_{0}$ is thus calculated as $m_{0}=1.354 \times 10^{3} \mathrm{~kg}$. The nominal (rated) rotational speed of the rotor is $\Omega_{0}=1.27 \mathrm{rad} / \mathrm{s}$.

\subsection{Dynamic amplification}

Figure 3 shows the dynamic amplification of the TMDI-controlled structural response (as a function of the normalized frequency $\left.\omega / \omega_{0}\right)$, where the optimal frequency-tuning formula Eq. (27) is used. The damper is located at a position, $x_{0}, 45 \mathrm{~m}$ along the blade from the blade root. The mass ratio $\mu$ of the TMDI is $5 \%$, and the inertance ratio $\beta$ is $5 \%$. Two different rotational speeds have been considered, i.e. $\Omega=0$ and $\Omega=\Omega_{0}$, corresponding to parked condition 
(a)

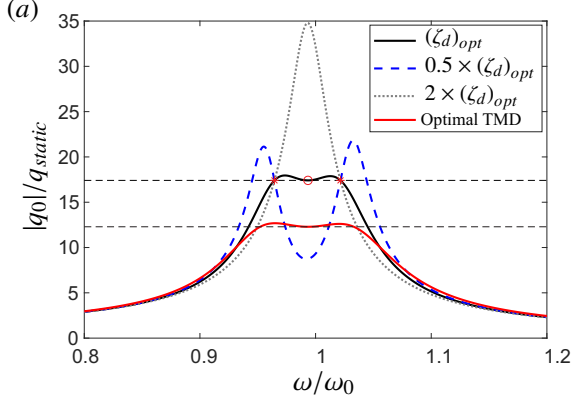

(b)

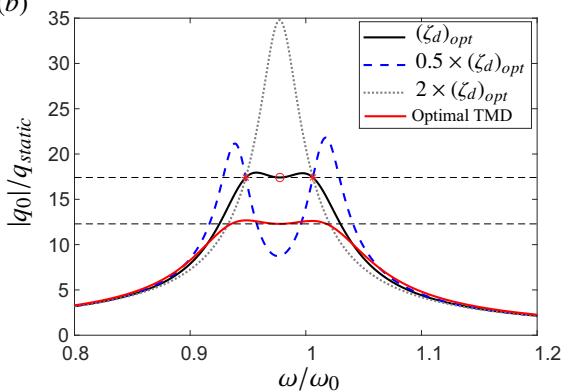

Figure 3: Dynamic amplification of the structural response for $x_{0}=45 \mathrm{~m}, \mu=0.05\left(m_{d}=67.7 \mathrm{~kg}\right)$ and $\beta=0.05$ $\left(b_{d}=67.7 \mathrm{~kg}\right)$. Asterisk: the fixed-point frequencies $\omega_{M}$ and $\omega_{N}$. Circle: $\omega_{\infty}$. (a) $\Omega=0$ (non-rotating blade). (b) $\Omega=\Omega_{0}(1.27 \mathrm{rad} / \mathrm{s})$

and normal operational condition of the rotor, respectively. For each $\Omega$, three different damping ratios have been considered, i.e. $\zeta_{\text {opt }}$ given by Eq. (32), $0.5 \times \zeta_{\text {opt }}$ (representing under-optimal damping) and $2 \times \zeta_{\text {opt }}$ (representing over-optimal damping). We can see that regardless of $\zeta_{d}$ and $\Omega$, the dynamic amplification at the two fixed-point frequencies $\omega_{M}$ and $\omega_{N}$ are always equal, which is in accordance with Eq. (30). When $\zeta_{\text {opt }}$ is used, the dynamic amplification at $\omega_{\infty}$ is identical to that at $\omega_{M}$ and $\omega_{N}$, resulting in a fairly flat plateau of the curve. On the other hand, the curve with under-optimal damping shows two significant peaks and one trough, and the curve with over-optimal damping has a very large peak at $\omega_{\infty}$. Comparing Figure 3(a) with (b), similar observations are made, except that the curves move towards left when $\Omega$ is increased from 0 to $\Omega_{0}$.

Furthermore, the results corresponding to an optimal classical TMD (tuned by Eqs. (28) and (33)) with the same mass ratio $\mu=0.05$ as the TMDI are also included in Figure 3 . It is seen that the optimal TMD outperforms the optimal TMDI in terms of the lowered dynamic amplification within a certain frequency interval. This makes sense because the inclusion of the inerter actually constrains the motion of the TMD mass (the absorber), thus reducing the energy absorption by the TMD mass. Therefore, the TMDI performs slightly worse than the classical TMD in damping blade vibrations.
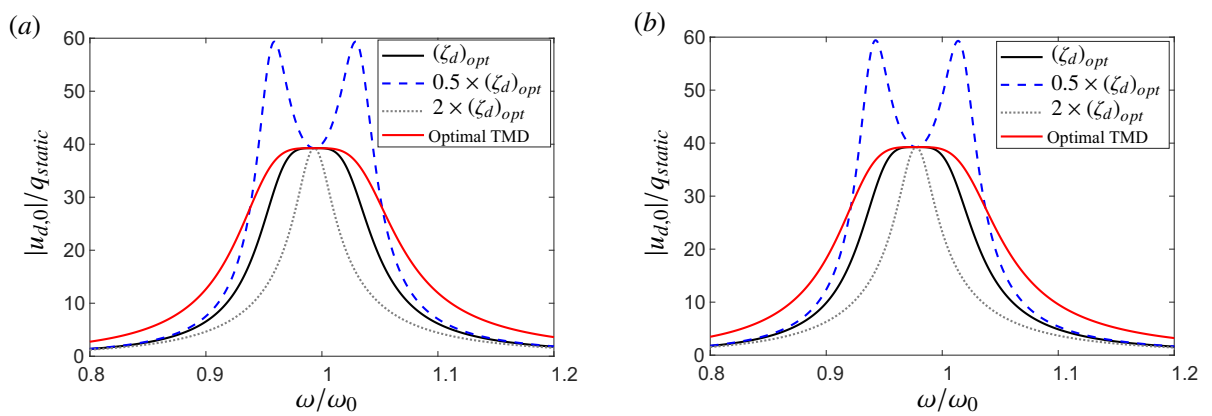

Figure 4: Dynamic amplification of the relative TMD block motion (the damper stroke) for $x_{0}=45 \mathrm{~m}, \mu=0.05$ ( $m_{d}=67.7 \mathrm{~kg}$ ) and $\beta=0.05\left(b_{d}=67.7 \mathrm{~kg}\right.$ ). (a) $\Omega=0$ (non-rotating blade). (b) $\Omega=\Omega_{0}(1.27 \mathrm{rad} / \mathrm{s})$.

In order to reveal the benefit of the proposed TMDI, Figure 4 shows the dynamic amplifica- 
tion of the relative TMD block motion (the damper stroke), with the same parameters as used in Figure 3. Again, three different damping ratios of the TMDI have been considered, and $\zeta_{\text {opt }}$ leads to the most flat behavior of the dynamic amplification of the damper stroke. The results of the optimal classical TMD are also shown in Figure 4. By comparison, it is observed that the curve corresponding to the optimal classical TMD is above the curve corresponding to the optimal TMDI for all frequencies, except at one frequency where the two curves coincide. This implies that the damper stroke of the TMDI is notably reduced comparing with that of TMD, which is the main advantage of the TMDI. Similar to the observations in Figure 3, the curves slightly moves towards left as $\Omega$ increases from 0 to $\Omega_{0}$.

\subsection{Influence of the inertance ratio and the detuning effect due to the change of rotational speed}

More in-depth parametric study is performed in this subsection. First, the influence of mass ratio $\mu$ and inertance ratio $\beta$ on the control effect and damper stroke of the TMDI are illustrated. Next, the detuning effects due to the change of rotational speed $\Omega$ are investigated.
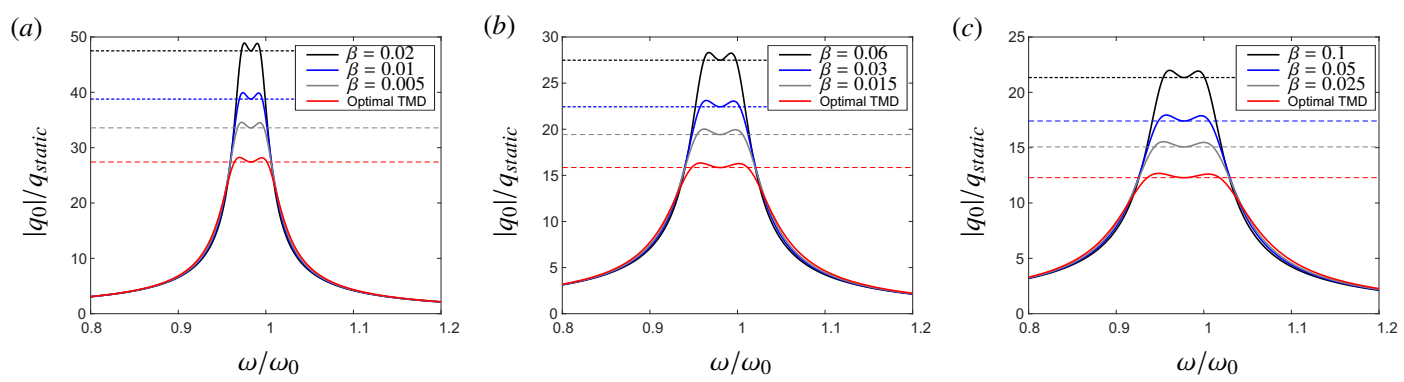

Figure 5: Influence of the mass ratio $\mu$ and inertance ratio $\beta$ on the vibration control effect of the TMDI for a rotating blade, $x_{0}=45 \mathrm{~m}, \Omega=\Omega_{0}(1.27 \mathrm{rad} / \mathrm{s})$, TMDI optimized. For a given $\mu$, three values of $\gamma$ are considered $(\gamma=2, \gamma=1$, $\gamma=0.5$ ). (a) $\mu=0.01$. (b) $\mu=0.03$. (c) $\mu=0.05$.

Figure 5 shows the influence of mass ratio $\mu$ and inertance ratio $\beta$ on the vibration control effect of the TMDI. $\mu=0.01, \mu=0.03$ and $\mu=0.05$ have been considered, as shown in Figure 5(a), (b) and (c), respectively. These correspond to the absorber mass $m_{d}=13.5 \mathrm{~kg}, m_{d}=40.6 \mathrm{~kg}$ and $m_{d}=67.7 \mathrm{~kg}$, respectively. For a given $\mu$, three different values of $\gamma\left(\gamma=\frac{\beta}{\mu}\right)$ have been considred, i.e. $\gamma=2, \gamma=1$ and $\gamma=0.5$. The TMDI is always optimized using Eqs. (27) and (32), and the results of the optimized TMD are also ploted for comparison. As $\mu$ increases (with fixed $\gamma$ ), the dynamic amplification of the structural response becomes lower and more broad, indicating a better control effect of the TMDI as expected. For a given mass ratio $\mu$, the increase of $\gamma$ (thus $\beta$ ) leads to larger dynamic amplification, implying worse vibration control effect. This is again explained by the constraint effect from the inerter on the absorber motion. The larger the inertance ratio is, the more significant constraint effect is exerted to the absorber motion, thus further reducing the energy absorption by the TMD mass. Therefore, the classic TMD with the same mass ratio always outperforms the TMDIs. For each given $\beta$, a dashed horizontal line calculated using Eq. (30) is also plotted, which passes through the two fixed-point frequencies and $\omega_{\infty}$. Therefore, Eq. (30) can be explicitly used as a performance indicator of vibration control effect of the blade-mounted TMDI when $\mu$ and $\beta$ are chosen.

Figure 6 shows the corresponding results of the damper stroke. As $\mu$ increases with fixed $\gamma$, the dynamic amplification of the damper stroke becomes significantly lower and more broad, 

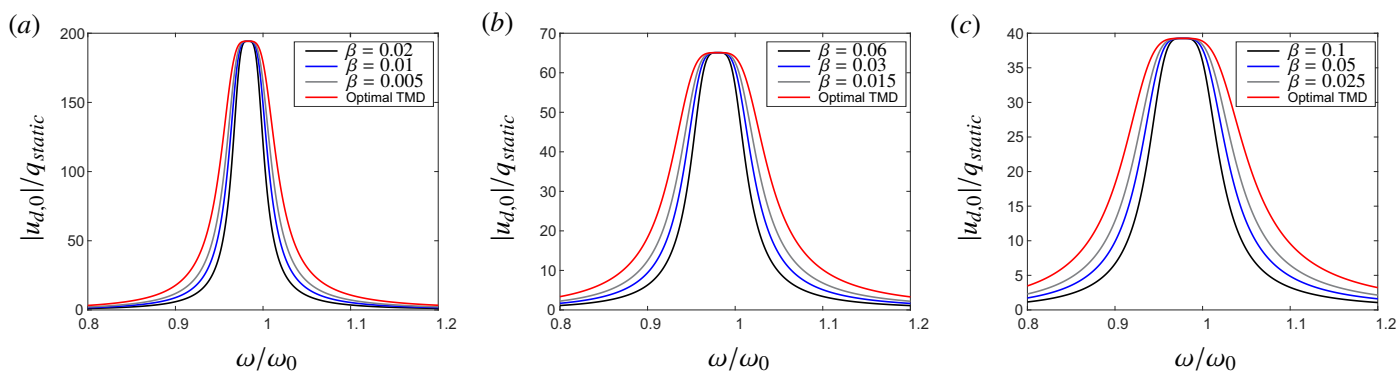

Figure 6: Influence of the mass ratio $\mu$ and inertance ratio $\beta$ on the damper stroke (installed on a rotating blade), $x_{0}=45$ $\mathrm{m}, \Omega=\Omega_{0}(1.27 \mathrm{rad} / \mathrm{s})$, TMDI optimized. For a given $\mu$, three values of $\gamma$ considered $(\gamma=2, \gamma=1, \gamma=0.5)$. (a) $\mu=0.01$. (b) $\mu=0.03$. (c) $\mu=0.05$.

which is again as expected. For a given $\mu$, the increase of $\gamma$ (and thus $\beta$ ) leads to much more narrow-banded curve, although the peak value turns out to be unchanged. This implies a smaller area below the curve as $\beta$ increases, and thus the damper stroke (as integration of contributions from all frequencies) decreases as $\beta$ increases. The damper stroke of the optimal TMD (with the same mass ratio $\mu$ ) always acts as the upper limit, i.e. the area below the curve corresponding to the optimal TMD is always larger than that of TMDI with $\beta>0$. Results in Figure 5 and 6 indicate that the increase of $\mu$ always results in a better TMDI performance in terms of both the control effect and the damper stroke (although $\mu$ should be limited due to practical considerations), as already well-known for classical vibration absorbers. On the other hand, the increase of $\beta$ leads to improved (reduced) damper stroke at the cost of worse control effect, and hence it is a tradeoff problem when choosing $\beta$.

Eqs. (27) and (32) clearly indicate that the optimal TMDI parameters depend explicitly on the rotor rotational speed $\Omega$. Hence it is of interest to investigate the performance of a passive TMDI tuned to non-rotating blade $(\Omega=0)$ on a rotating blade, i.e. the detuning effect due to change of $\Omega$.
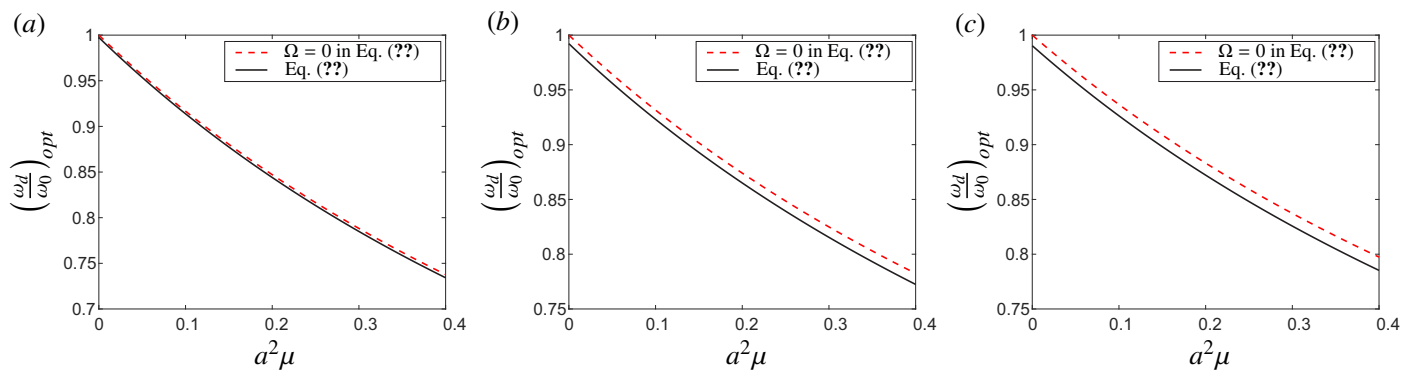

Figure 7: Optimal frequency-tuning ratio as a function of the non-dimensional parameter $a^{2} \mu, \Omega=\Omega_{0}(1.27 \mathrm{rad} / \mathrm{s})$. (a) $\beta=0.01$. (b) $\beta=0.05$. (c) $\beta=0.08$.

Figure 7 shows the optimal frequency-tuning ratio $\left(\frac{\omega_{d}}{\omega_{0}}\right)_{\text {opt }}$ as a function of $a^{2} \mu$, using Eq. (27) or Eq. (27) with $\Omega=0$, for a rotating blade with $\Omega=\Omega_{0}$. Three different values of $\beta$ have been considered. It is observed that the deviation between the two curves increases as $\beta$ increases. Actually, when $\beta=0$ (thus $\gamma=0$ ), Eq. (27) is reduced to Eq. (28) which is independent on $\Omega$. 
For a given $\beta$, the deviation of the two curves increases slightly as $a^{2} \mu$ increases. Therefore, when large $\mu$ and $\beta$ are chosen for the TMDI installed in a rotating blade, significant frequency-tuning error will be introduced by tuning the TMDI to a non-rotating blade.
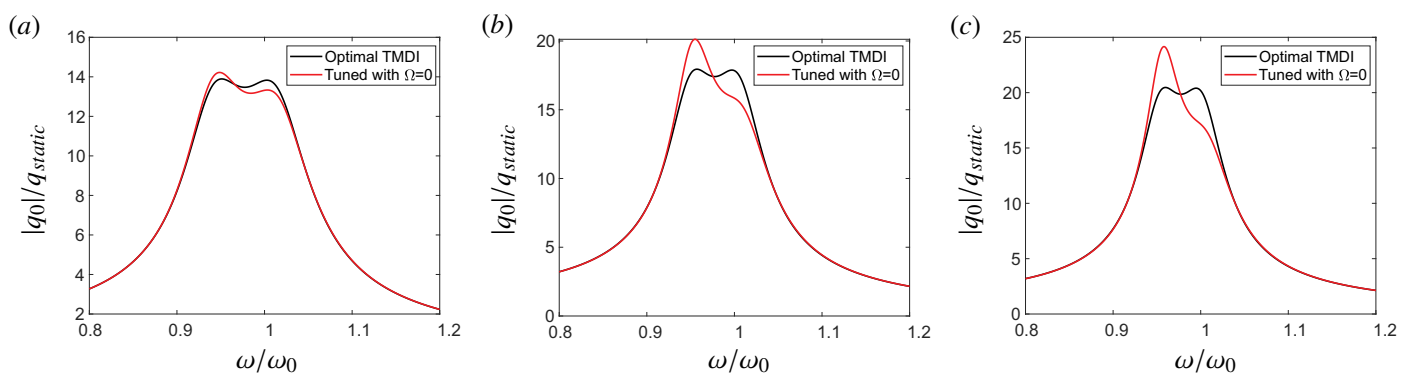

Figure 8: Performance (dynamic amplification of the structural response) comparison when using the optimal TMDI (Eqs. (27) and (32)) and the TMDI tuned by assuming $\Omega=0$ in a rotating blade. $\Omega=\Omega_{0}(1.27 \mathrm{rad} / \mathrm{s}), x_{0}=45 \mathrm{~m}$, $\mu=0.05$. (a) $\beta=0.01$. (b) $\beta=0.05$. (c) $\beta=0.08$.

Figure 8 compares the dynamic amplification of the structural response of the optimal TMDI (Eq. (27) for frequency tuning and Eq. (32) for damping tuning) and the non-optimal TMDI (tuned by assuming $\Omega=0$ ), for a rotating blade. As seen in Figure 8(a), the difference is insignificant when $\beta=0.01$, in accordance with the observation in Figure 7(a). As $\beta$ increases, the error becomes much more noticeable as shown in Figure 8(b) and (c). The optimal TMDI leads to a completely flat plateau of the curve, while the non-optimal TMDI leads to a skewed curve with higher amplitude to the left, exhibiting a much higher maximum amplitude of the structural response. This detunnig effect becomes very significant for $\beta=0.08$. Therefore, semiactive control strategy is needed for the TMDI with large $\beta$, so that the TMDI parameters can be adjusted according to the rotor rotational speed.
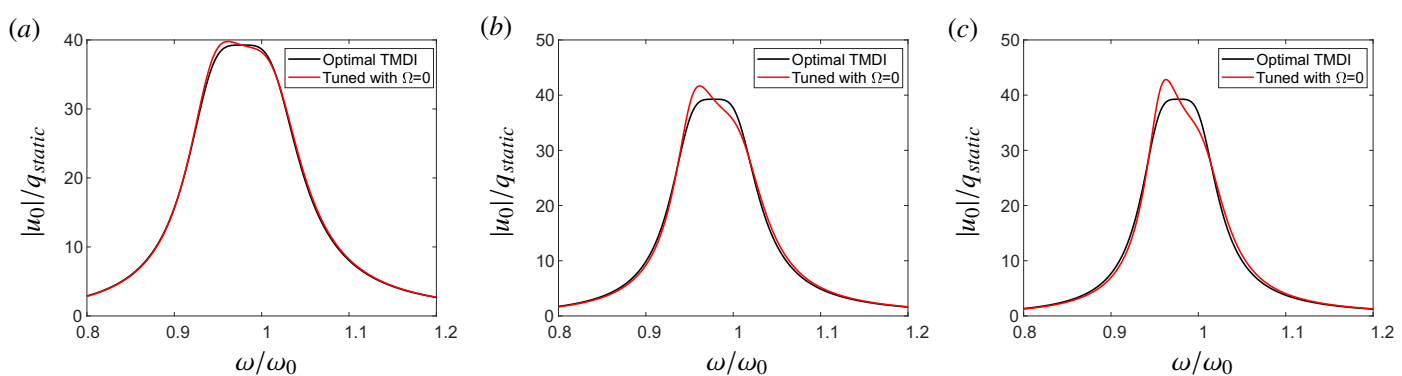

Figure 9: Performance (dynamic amplification of the relative TMD block motion) comparison when using the optimal TMDI (Eqs. (27) and (32)) and the TMDI tuned by assuming $\Omega=0$ in a rotating blade. $\Omega=\Omega_{0}(1.27 \mathrm{rad} / \mathrm{s}), x_{0}=45 \mathrm{~m}$, $\mu=0.05$. (a) $\beta=0.01$. (b) $\beta=0.05$. (c) $\beta=0.08$.

Figure 9 shows the corresponding results of the damper stroke. Similar observations can be made as in Figure 8, although the detuning effect is less significantly revealed in the damper stroke. It should also be noted that all the above results are based on the NREL $5 \mathrm{MW}$ wind turbine with its rotational speed ranging between 0 and $\Omega_{0}$, and for other types of wind turbines with larger variations of rotor rotational speed, the detuning effect by tuning the TMDI to a non-rotating blade might be more significant. 


\subsection{Time-domain simulation}

(a)

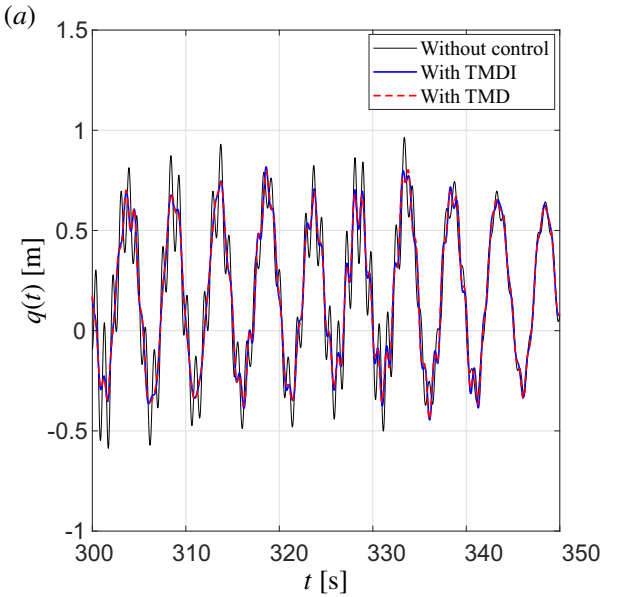

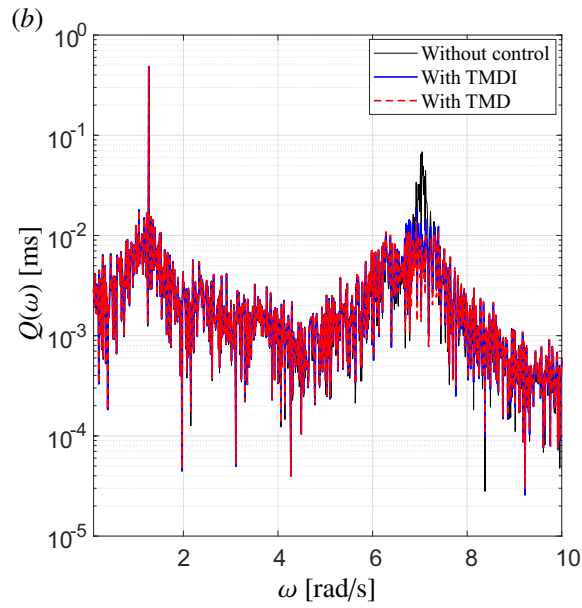

Figure 10: Blade edgewise vibrations without control, with TMDI and with TMD. Normal operational conditions $\left(\zeta_{0}=\right.$ 0.005). $x_{0}=45 \mathrm{~m}, \mu=0.05$ and $\beta=0.05$. (a) Time series. (b) Fourier amplitude in semi-logarithmic chart.

(a)

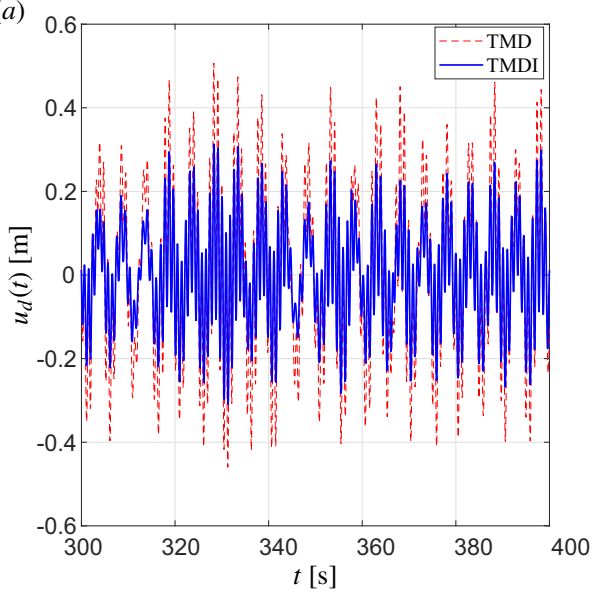

(b)

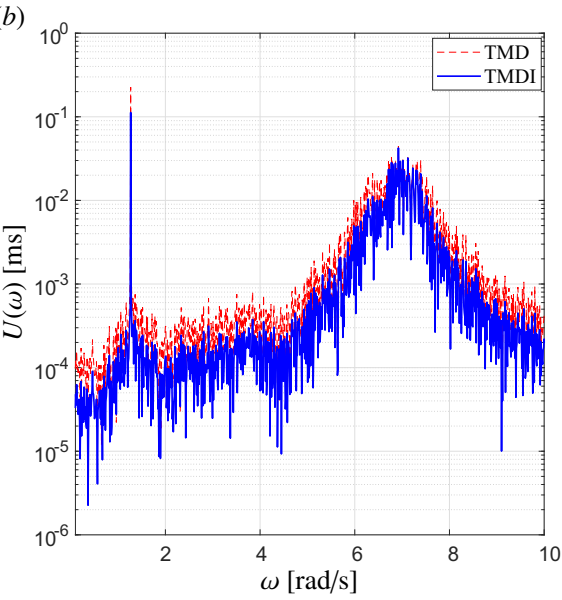

Figure 11: Stroke of the dampers. Normal operational conditions $\left(\zeta_{0}=0.005\right) . x_{0}=45 \mathrm{~m}, \mu=0.05$ and $\beta=0.05$. (a) Time series. (b) Fourier amplitude in semi-logarithmic chart.

In this subsection, the performance of the TMDI and the TMD are compared in the time domain by solving Eq. (13). Figure 10 (a) shows the blade edgewise vibration time history under normal operational conditions with overall damping $\zeta_{0}=0.005$. Both the optimal TMDI and the optimal TMD are used, and same values of $x_{0}, \mu$ and $\beta$ as in Figure 3 are employed. From the time history response it is clear that the TMD and TMDI can mitigate the edgewise vibrations of the turbine blade. Peak response reductions and peak-to-peak reductions of $19 \%$ are achieved by the dampers. From these numerical simulations it appears that the proposed TMDI configuration performs very slightly worse than the TMD (with the same mass ratio) when 
vibration suppression is considered. The plot of the Fourier amplitude of the blade responses in Figure 10 (b) confirms this time-history behaviour. Two peaks are observed in the frequency domain plot. The first corresponds to the rotational speed of the blade, $\Omega=1.26 \mathrm{rad} / \mathrm{s}$. The second peak corresponds to the edgewise natural frequency of the blade which is $6.7 \mathrm{rad} / \mathrm{s}$. Both the TMD and TMDI are successful at suppressing this second peak with the TMD performing slightly better from a vibration control perspective. However, the benefit of the TMDI is evident when damper stroke is considered. Figure 11 (a) shows a time history plot of the damper strokes under normal operating conditions. From this plot is clear that the relative motion of the damper mass becomes smaller when the inerter is added, i.e. the mass block in the TMDI has smaller stroke than that of the mass block in the TMD. The stroke of the damper is reduced by up to $40 \%$ due to the addition of the inerter.
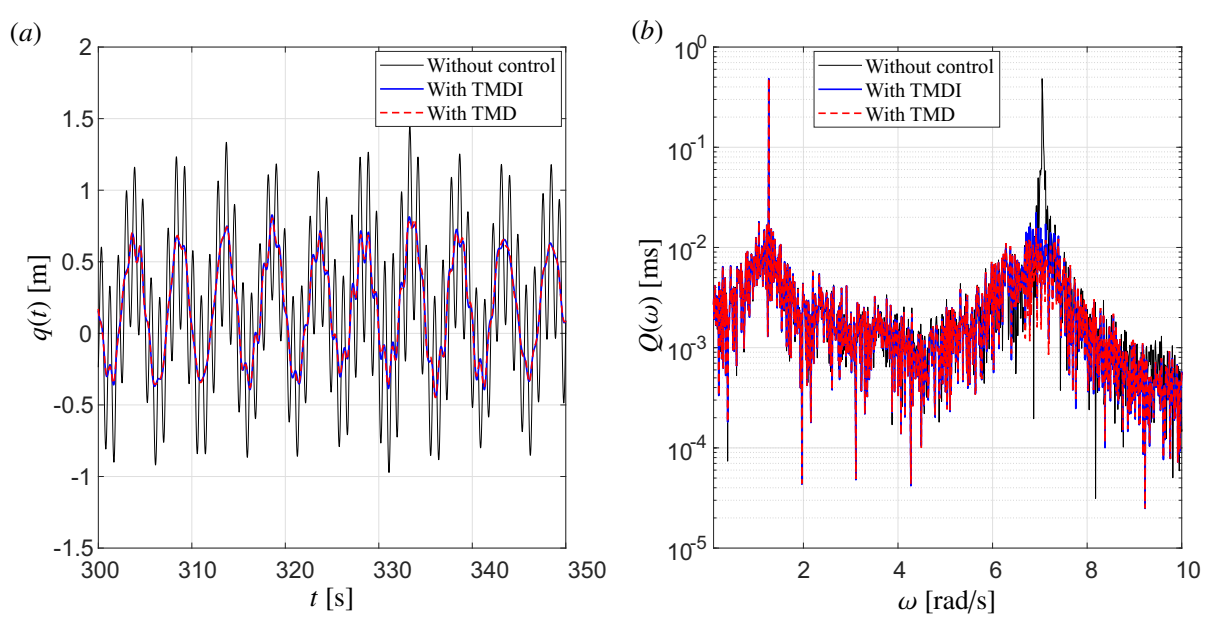

Figure 12: Blade edgewise vibrations without control, with TMDI and with TMD. Total damping $\zeta_{0}=0 . x_{0}=45 \mathrm{~m}$, $\mu=0.05$ and $\beta=0.05$. (a) Time series. (b) Fourier amplitude in semi-logarithmic chart.

A further numerical example is considered with zero blade damping. When considering edgewise vibrations it is important to check this case due to the fact that the blade is very lightly damped in the edgewise direction. There is also the possibility of negative aerodynamic damping in this direction. As a result, the sum of the structural damping and the aerodynamic damping can be less than zero [60]. For these reasons in design situations edgewise blade damping is often ignored. Figure 12 (a) shows the blade edgewise vibration time history with total damping $\zeta_{0}, x_{0}=45 \mathrm{~m}, \mu=5 \%$ and $\beta=5 \%$. The dampers display excellent performance in this case with peak vibration reductions of 50\% and peak-to-peak reductions of 55\%. The frequency plot in Figure 12 (b) confirms this behaviour and again shows the slightly worse performance of the TMDI with respect to vibration reduction. Figure 13 (a) shows the damper stroke time history plot for the case of zero blade damping. The TMDI again has a considerably smaller stroke requirement than a TMD of the same mass ratio. The TMDI stroke is up to 50\% smaller than the TMD stroke.

Finally, a new scenario where the dampers are mounted at a location closer to the blade tip $\left(x_{0}=55 \mathrm{~m}\right)$ is considered, to further illustrate the benefit of the TMDI in terms of damper stroke reduction. The vibration frequency corresponding to the edgewise blade mode of floating offshore wind turbines is normally low, in this case for a 5MW turbine it is approximately 1 

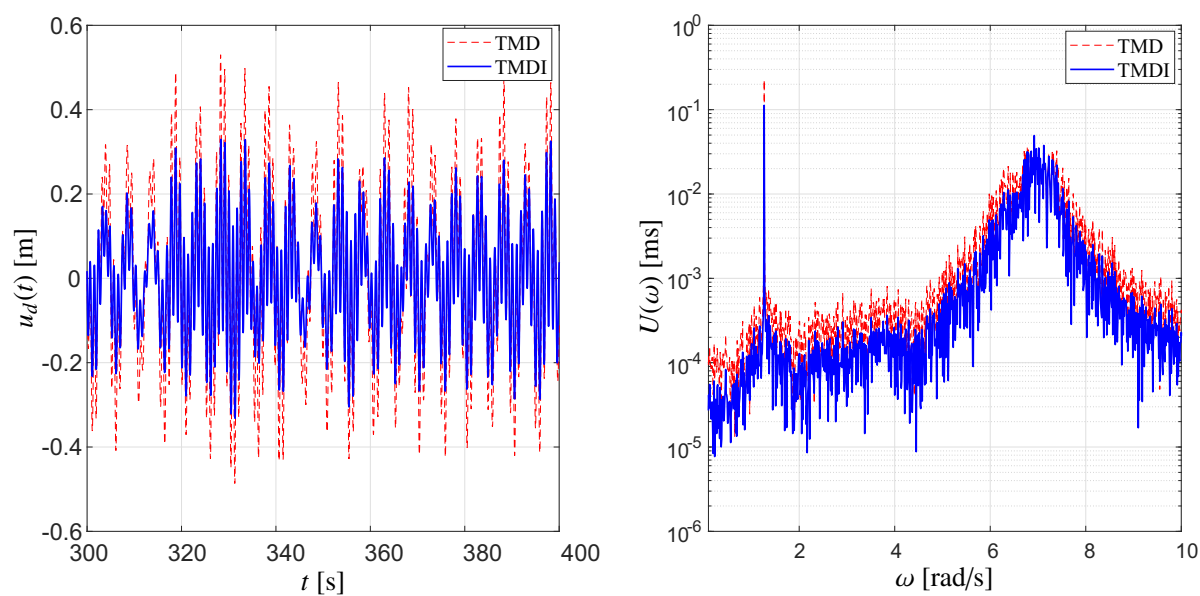

Figure 13: Stroke of the dampers. Total damping $\zeta_{0}=0 . x_{0}=45 \mathrm{~m}, \mu=0.05$ and $\beta=0.05$. (a) Time series. (b) Fourier amplitude in semi-logarithmic chart.

Hz. A reasonable stroke is thus required when a damper is applied to control these responses. This may not be practical due to the limited installation space available inside the blade. To be most effective the damper should be located as close to the blade tip as possible. However, this is the location with the least available space inside the blade to accommodate a damper. Table 1 provides details on the chord length inside the blade at various locations $x_{0}$ and the blade thickness, i.e. the maximum distance between upper and lower blade surfaces, at these locations. It should be noted that the available damper stroke will be significantly less than the chord length depending on the dimensions of the damper mass and the thickness.

\begin{tabular}{ccc}
\hline$x_{0}(\mathrm{~m})$ & Chord length $(\mathrm{m})$ & Thickness $(\mathrm{m})$ \\
20.0 & 4.5 & 1.60 \\
30.0 & 3.9 & 1.00 \\
45.0 & 3.0 & 0.55 \\
55.0 & 2.3 & 0.42 \\
61.6 & 1.4 & 0.25 \\
\hline
\end{tabular}

Table 1: Chord lengths and thicknesses of NREL 5MW wind turbine blade [57, 61]

In this case, the mass ratio of the dampers is reduced to $\mu=0.03$, and the inertance of the TMDI is increased to $\beta=0.08$. Figure 14 (a) shows the time series of the blade edgewise vibrations. The behaviour of the blade controlled by a TMDI is very similar in nature to a blade controlled by a traditional TMD with good vibration reductions observed in both cases. Figure 14 (b) shows the frequency domain information. It is evident from this that there is a slight degradation in vibration control performance when the TMDI is used - however, this is not perceptible from the time series. Figure 14 (c) shows the time series of the damper strokes. In this case the stroke reduction effect of the TMDI is more significant than in the previous cases studied. The TMDI has significantly less stroke requirement than the TMD. The TMDI stroke is reduced by more than $55 \%$ compared to the TMD stroke. Figure 14 (d) confirms this stroke reduction in the frequency domain. 

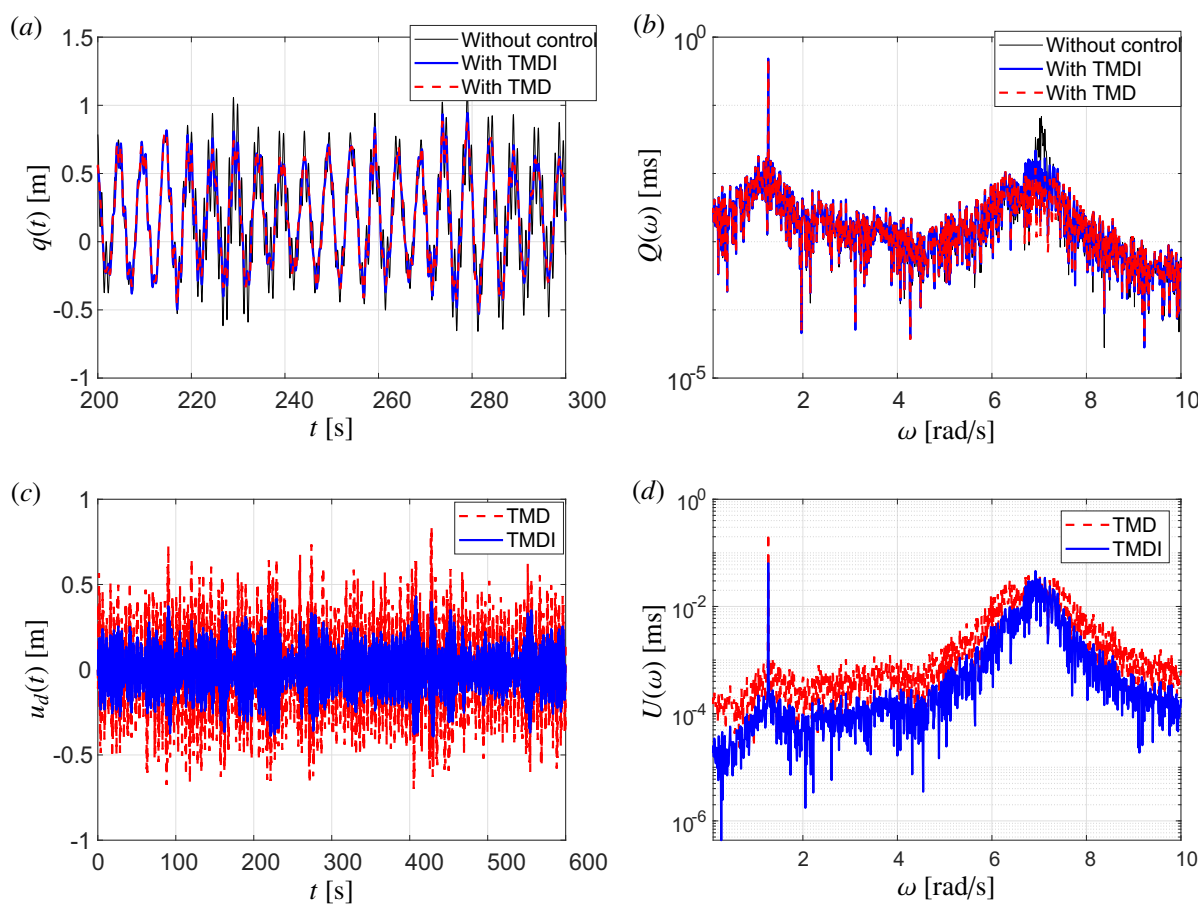

Figure 14: Performance of the TMDI when mounted closer to blade tip. Total damping $\zeta_{0}=0.005$. $x_{0}=55 \mathrm{~m}$, $\mu=0.03$ and $\beta=0.08$. (a) Time series of blade edgewise vibrations. (b) Fourier amplitude of the blade edgewise vibrations in semi-logarithmic chart. (c) Time series of the damper strokes. (d) Fourier amplitude of the damper strokes in semi-logarithmic chart.

This is a very significant reduction in damper stroke and has some very practical benefits. At the location $x_{0}=55 \mathrm{~m}$ the chord length inside the blade is $2.3 \mathrm{~m}$ and the blade thickness is $0.42 \mathrm{~m}$. The available stroke would therefore be significantly less than the chord length. A traditional TMD would require stroke of up to $1.5 \mathrm{~m}$ versus stroke of $0.7 \mathrm{~m}$ for the TMDI, see Figure 14 (c). A traditional TMD would probably not be viable at this location due to the large stroke requirement. Using a TMDI at this location allows one to take advantage of the greater vibration mitigation due to closer proximity to the blade tip, while ensuring that the damper stroke can be accommodated in the blade.

\subsection{Evaluation by the 13-DOF aeroelastic model}

To verify the applicability of the closed-form optimization for TMDI tuning (Section 3) and the performance of the damper in a highly coupled wind turbine system, the optimized TMDI is incorporated into the 13-DOF model. For each blade, a TMDI is installed at the position of $x_{0}=45 \mathrm{~m}$, resulting in a 16-DOF system for the wind turbine with totally three dampers installed. The equations of motion of the wind turbine system is extended by means of Eq. (13), and are rewritten in the state-space form. Including the first-order filter equation for the pitch controller, the dynamics of the coupled system is described by a 33-dimensional state vector, and can be solved using the fourth-order Runge-Kutta method.

Figure 15 shows similar behaviour as in Figures 10 and 11 where the same damper parameters 
(a)

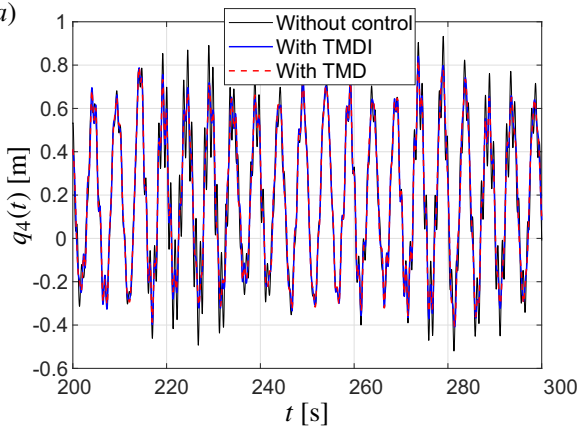

(c)

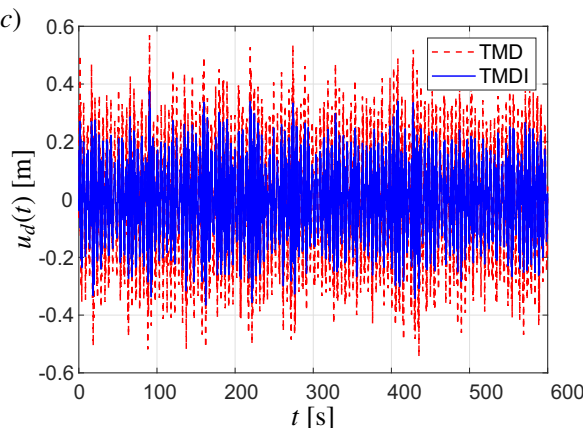

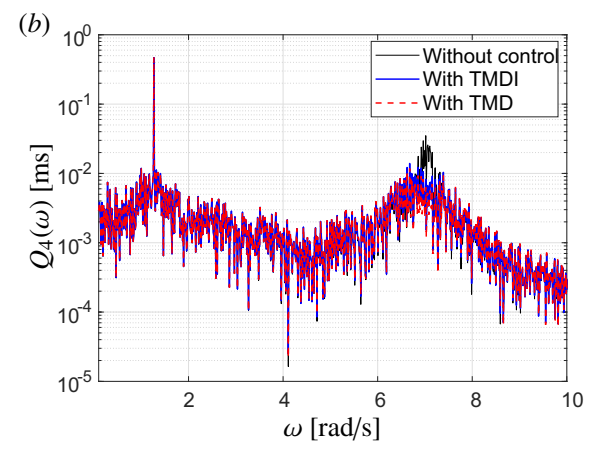

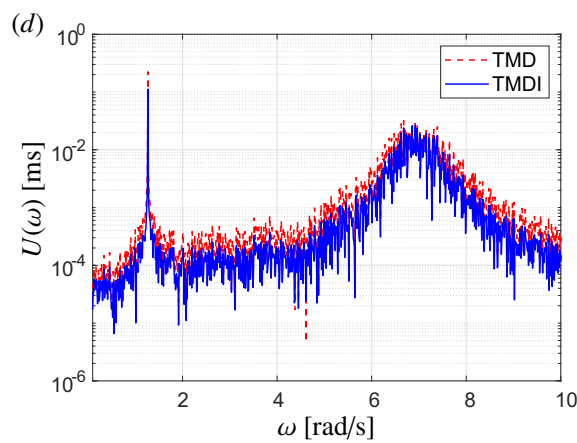

Figure 15: Performance of the TMDI evaluated using the fully-coupled 13-DOF aeroelastic model. Structural damping ratio of the blade: $0.005 . x_{0}=45 \mathrm{~m}, \mu=0.05$ and $\beta=0.05$. (a) Time series of blade edgewise vibrations $q_{4}(t)$ of the 13-DOF model. (b) Fourier amplitude of the blade edgewise vibrations $q_{4}(t)$ in semi-logarithmic chart. (c) Time series of the damper strokes. (d) Fourier amplitude of the damper strokes in semi-logarithmic chart. 
were used in a reduced order model. There is a slight degradation in blade vibration control noticeable in the frequency domain (Figure 15 (b)). However, the damper stroke required by the TMDI is substantially less than the stroke required by a traditional TMD (over $50 \%$ in some cases) as confirmed by time series results (Figure 15 (c)) and in the frequency domain (Figure 15 (d)).

\section{Conclusions}

This paper proposed a novel TMDI for vibration control of offshore wind turbine blades. A multi degree of freedom offshore wind turbine model was developed with TMDIs incorporated into the hollow wind turbine blades. Closed-form expressions have been derived for the optimal tuning and damping ratios of the TMDI. Numerical simulations were performed to determine the effectiveness of the new damper in controlling edgewise vibrations. The inclusion of the inerter in the damper had a significant effect on the damper stroke. The inerter reduced the stroke of the damper by up to 55\%. The results clearly show that the TMDI improves the dynamic response of the blades. The damper significantly reduces blade vibrations. The fundamental compromise in using the TMDI over a more traditional TMD is evident. It is possible to greatly reduce the damper stroke at the cost of very slightly increased blade vibration. Reductions in the damper stroke of over $50 \%$ can be achieved with small degradations in blade vibration control performance. This is a trade-off that must be considered in the design of a wind turbine. However, it should be noted that the vibration control performance reduction is very slight and hardly noticeable in the time domain. Furthermore, since the space available for stroke within wind turbine blades is very limited, the reduction in the damper stroke is very beneficial and demonstrates a very practical advantage over classical TMDs for wind turbine blade applications.

\section{Acknowledgments}

The supports of Aarhus University Research Foundation under the AUFF Assistant Professor Starting Grant (AUFF-E-2017-7-20), the Committee of Science and Technology of Shanghai China (Grant No. 18160712800) and the Sustainable Energy Authority of Ireland (Grant No. RDD/213) are highly appreciated.

\section{References}

[1] Kwok, K., Samali, B.. Performance of tuned mass dampers under wind loads. Engineering Structures 1995;17(9):655-667.

[2] Sadek, F., Mohraz, B., Taylor, A.W., Chung, R.M.. A method of estimating the parameters of tuned mass dampers for seismic applications. Earthquake Engineering \& Structural Dynamics 1997;26(6):617-635.

[3] Suhardjo, J., Kareem, A.. Feedback-feedforward control of offshore platforms under random waves. Earthquake Engineering \& Structural Dynamics 2001;30(2):213-235. URL: $\quad$ http://dx.doi.org/10.1002/1096-9845(200102) 30:2<213: :AID-EQE5>3.0.CO;2-4. doi:10.1002/1096-9845(200102)30:2¡213::AID-EQE5;3.0.CO;2-4.

[4] Nielsen, S.R.K.. Vibration Theory: Linear vibration theory. Aalborg tekniske Universitetsforlag; 2004.

[5] Frahm, H.. Device for damping vibrations of bodies. 1911. US Patent 989,958.

[6] Ormondroyd, J., DenHartog, J.. The theory of the dynamic vibration absorber. Journal of Applied Mechanics, Transactions of the American Society of Mechanical Engineers 1928;:4950.

[7] DenHartog, J.. Mechanical Vibrations, 4th Edition. McGraw-Hill, New York; 1956.

[8] Fujino, Y., Abé, M.. Design formulas for tuned mass dampers based on a perturbation technique. Earthquake Engineering \& Structural Dynamics 1993;22(10):833-854. URL: http: //dx.doi .org/10. 1002/eqe. 4290221002. doi: $10.1002 /$ eqe.4290221002. 
[9] Rana, R., Soong, T.. Parametric study and simplified design of tuned mass dampers. Engineering Structures 1998;20(3):193 - $204 . \quad$ URL: http://www.sciencedirect.com/science/article/pii/S0141029697000783. doi:10.1016/S01410296(97)00078-3; ; ce:title $\underset{i}{ }$ Structural Controli/ce:title $_{i}$.

[10] Ghosh, A., Basu, B.. A closed-form optimal tuning criterion for tmd in damped structures. Structural Control and Health Monitoring 2007;14(4):681-692. URL: http://dx.doi .org/10.1002/stc. 176. doi:10.1002/stc.176.

[11] Krenk, S.. Frequency analysis of the tuned mass damper. Journal of applied mechanics 2005;72(6):936-942.

[12] Smith, M.C.. Synthesis of mechanical networks: the inerter. In: Decision and Control, 2002, Proceedings of the 41st IEEE Conference on; vol. 2. IEEE; 2002, p. 1657-1662.

[13] Gonzalez-Buelga, A., Lazar, I.F., Jiang, J.Z., Neild, S.A., Inman, D.J.. Assessing the effect of nonlinearities on the performance of a tuned inerter damper. Structural Control and Health Monitoring 2017;24(3):e1879.

[14] Swift, S., Smith, M.C., Glover, A., Papageorgiou, C., Gartner, B., Houghton, N.E.. Design and modelling of a fluid inerter. International Journal of Control 2013;86(11):2035-2051.

[15] De Domenico, D., Deastra, P., Ricciardi, G., Sims, N.D., Wagg, D.J.. Novel fluid inerter based tuned mass dampers for optimised structural control of base-isolated buildings. Journal of the Franklin Institute 2019;356(14):7626-7649.

[16] De Domenico, D., Ricciardi, G.. An enhanced base isolation system equipped with optimal tuned mass damper inerter (tmdi). Earthquake engineering \& structural dynamics 2018;47(5):1169-1192.

[17] De Domenico, D., Ricciardi, G.. Improving the dynamic performance of base-isolated structures via tuned mass damper and inerter devices: A comparative study. Structural Control and Health Monitoring 2018;25(10):e2234.

[18] Pietrosanti, D., De Angelis, M., Basili, M.. Optimal design and performance evaluation of systems with tuned mass damper inerter (tmdi). Earthquake Engineering \& Structural Dynamics 2017;46(8):1367-1388.

[19] De Domenico, D., Ricciardi, G.. Optimal design and seismic performance of tuned mass damper inerter (tmdi) for structures with nonlinear base isolation systems. Earthquake engineering \& structural dynamics 2018;47(12):2539_ 2560 .

[20] Ikago, K., Saito, K., Inoue, N.. Seismic control of single-degree-of-freedom structure using tuned viscous mass damper. Earthquake Engineering \& Structural Dynamics 2012;41(3):453-474.

[21] Garrido, H., Curadelli, O., Ambrosini, D.. Improvement of tuned mass damper by using rotational inertia through tuned viscous mass damper. Engineering Structures 2013;56:2149-2153.

[22] Marian, L., Giaralis, A.. Optimal design of a novel tuned mass-damper-inerter (tmdi) passive vibration control configuration for stochastically support-excited structural systems. Probabilistic Engineering Mechanics 2014;38:156164.

[23] Giaralis, A., Taflanidis, A.A.. Reliability-based design of tuned mass-damper-inerter (tmdi) equipped multistorey frame buildings under seismic excitation. In: 12th International Conference on Applications of Statistics and Probability in Civil Engineering, ICASP 2015. University of British Columbia Library; 2015,.

[24] Lazar, I., Neild, S., Wagg, D.. Using an inerter-based device for structural vibration suppression. Earthquake Engineering \& Structural Dynamics 2014;43(8):1129-1147.

[25] Lazar, I., Neild, S., Wagg, D.. Vibration suppression of cables using tuned inerter dampers. Engineering Structures 2016;122:62-71

[26] Hu, Y., Chen, M.Z.. Performance evaluation for inerter-based dynamic vibration absorbers. International Journal of Mechanical Sciences 2015;99:297-307.

[27] Shen, W., Niyitangamahoro, A., Feng, Z., Zhu, H.. Tuned inerter dampers for civil structures subjected to earthquake ground motions: optimum design and seismic performance. Engineering Structures 2019;198:109470.

[28] Colwell, S., Basu, $\quad$ B.. Tuned liquid column dampers in offshore wind turbines for structural control. Engineering Structures 2009;31(2):358 - 368 URL: http://www.sciencedirect.com/science/article/pii/S0141029608003076. doi:10.1016/j.engstruct.2008.09.001.

[29] Dueñas-Osorio, L., Basu, B.. Unavailability of wind turbines due to wind-induced accelerations. Engineering Structures 2008;30(4):885-893.

[30] Zhang, Z., Basu, B., Nielsen, S.R.. Tuned liquid column dampers for mitigation of edgewise vibrations in rotating wind turbine blades. Structural Control and Health Monitoring 2015;22(3):500-517.

[31] Zhang, Z., Nielsen, S.R., Basu, B., Li, J.. Nonlinear modeling of tuned liquid dampers (tlds) in rotating wind turbine blades for damping edgewise vibrations. Journal of Fluids and Structures 2015;59:252-269.

[32] Basu, B., Zhang, Z., Nielsen, S.R.. Damping of edgewise vibration in wind turbine blades by means of circular liquid dampers. Wind Energy 2016;19(2):213-226.

[33] Zhang, Z., Li, J., Nielsen, S.R., Basu, B.. Mitigation of edgewise vibrations in wind turbine blades by means of roller dampers. Journal of Sound and Vibration 2014;333(21):5283-5298.

[34] Murtagh, P.J., Ghosh, A., Basu, B., Broderick, B.M.. Passive control of wind turbine vibrations including blade/tower interaction and rotationally sampled turbulence. Wind Energy 2008;11(4):305-317. URL: 
http://dx.doi.org/10.1002/we.249. doi:10.1002/we.249.

[35] Lackner, M.A., Rotea, M.A.. Passive structural control of offshore wind turbines. Wind Energy 2011;14(3):373388. URL: http://dx.doi.org/10.1002/we.426. doi:10.1002/we.426.

[36] Zhang, Z., Staino, A., Basu, B., Nielsen, S.R.. Performance evaluation of full-scale tuned liquid dampers (tlds) for vibration control of large wind turbines using real-time hybrid testing. Engineering Structures 2016;126:417-431.

[37] Arrigan, J., Pakrashi, V., Basu, B., Nagarajaiah, S.. Control of flapwise vibrations in wind turbine blades using semi-active tuned mass dampers. Structural Control and Health Monitoring 2011;18(8):840-851. URL: http://dx.doi.org/10.1002/stc.404. doi:10.1002/stc.404.

[38] Arrigan, J., Huang, C., Staino, A., Basu, B., Nagarajaiah, S.. A frequency tracking semi-active algorithm for control of edgewise vibrations in wind turbine blades. Smart Structures and Systems 2014;13(2):177-201.

[39] Fitzgerald, B., Arrigan, J., Basu, B.. Damage detection in wind turbine blades using time-frequency analysis of vibration signals. In: Neural Networks (IJCNN), The 2010 International Joint Conference on. IEEE; 2010, p. 1-5.

[40] Fitzgerald, B., Basu, B., Nielsen, S.R.K.. Active tuned mass dampers for control of in-plane vibrations of wind turbine blades. Structural Control and Health Monitoring 2013;20(12):1377-1396. URL: http://dx.doi.org/10.1002/stc.1524. doi:10.1002/stc.1524.

[41] Fitzgerald, B., Sarkar, S., Staino, A.. Improved reliability of wind turbine towers with active tuned mass dampers (atmds). Journal of Sound and Vibration 2018;419:103-122.

[42] Lackner, M.A., Rotea, M.A.. Structural control of floating wind turbines. Mechatronics 2011;21(4):704 - 719. URL: http://www.sciencedirect.com/science/article/pii/S0957415810002072. doi:http://dx.doi.org/10.1016/j.mechatronics.2010.11.007.

[43] Fitzgerald, B., Basu, B.. Structural control of wind turbines with soil structure interaction included. Engineering Structures 2016;111:131-151.

[44] Staino, A., Basu, B., Nielsen, S.R.. Actuator control of edgewise vibrations in wind turbine blades. Journal of Sound and Vibration 2012;331(6):1233-1256.

[45] Fitzgerald, B., Basu, B.. Cable connected active tuned mass dampers for control of in-plane vibrations of wind turbine blades. Journal of Sound and Vibration 2014;333(23):5980-6004.

[46] Staino, A., Basu, B.. Emerging trends in vibration control of wind turbines: a focus on a dual control strategy. Philosophical Transactions of the Royal Society A: Mathematical, Physical and Engineering Sciences 2015;373(2035):20140069.

[47] Fitzgerald, B., Staino, A., Basu, B.. Wavelet-based individual blade pitch control for vibration control of wind turbine blades. Structural Control and Health Monitoring 2019;26(1):e2284.

[48] Sarkar, S., Fitzgerald, B., Basu, B.. Individual blade pitch control of floating offshore wind turbines for load mitigation and power regulation. IEEE Transactions on Control Systems Technology 2020;.

[49] Sarkar, S., Chen, L., Fitzgerald, B., Basu, B.. Multi-resolution wavelet pitch controller for spar-type floating offshore wind turbines including wave-current interactions. Journal of Sound and Vibration 2020;:115170.

[50] Hu, Y., Wang, J., Chen, M.Z., Li, Z., Sun, Y.. Load mitigation for a barge-type floating offshore wind turbine via inerter-based passive structural control. Engineering Structures 2018;177:198-209.

[51] Zhang, R., Zhao, Z., Dai, K.. Seismic response mitigation of a wind turbine tower using a tuned parallel inerter mass system. Engineering Structures 2019;180:29-39.

[52] Ma, R., Bi, K., Hao, H.. Mitigation of heave response of semi-submersible platform (ssp) using tuned heave plate inerter (thpi). Engineering Structures 2018;177:357-373.

[53] Ma, R., Bi, K., Hao, H.. A novel rotational inertia damper for heave motion suppression of semisubmersible platform in the shallow sea. Structural Control and Health Monitoring 2019;:e2368.

[54] Sarkar, S., Fitzgerald, B.. Vibration control of spar-type floating offshore wind turbine towers using a tuned mass-damper-inerter. Structural Control and Health Monitoring 2020;27(1):e2471.

[55] Zhang, Z., Nielsen, S., Blaabjerg, F., Zhou, D.. Dynamics and control of lateral tower vibrations in offshore wind turbines by means of active generator torque. Energies 2014;7(11):7746-7772.

[56] Svendsen, M.N., Krenk, S., Høgsberg, J.. Resonant vibration control of rotating beams. Journal of Sound and Vibration 2011;330(9):1877-1890.

[57] Jonkman, J., Butterfield, S., Musial, W., Scott, G.. Definition of a 5-mw reference wind turbine for offshore system development. Tech. Rep.; National Renewable Energy Lab.(NREL), Golden, CO (United States); 2009.

[58] Pars, L.A.. A treatise on analytical dynamics. Wiley; 1965.

[59] Zhang, Z.. Optimal tuning of the tuned mass damper (tmd) for rotating wind turbine blades. Engineering Structures 2020;207:110209.

[60] Thomsen, K., Petersen, J.T., Nim, E., Øye, S., Petersen, B.. A method for determination of damping for edgewise blade vibrations. Wind Energy: An International Journal for Progress and Applications in Wind Power Conversion Technology 2000;3(4):233-246.

[61] Resor, B.R.. Definition of a 5mw/61.5 m wind turbine blade reference model. Albuquerque, New Mexico, USA, Sandia National Laboratories, SAND2013-2569 2013;2013. 